\title{
Transit Network Sensitivity Analysis
}

\author{
Young-Jae Lee, Morgan State University
}

\begin{abstract}
Transit network conditions change everyday. While those changes should be considered for modifying a transit network, formulating and optimizing the whole transit network may be a costly, difficult task.

This article uses a developed transit network design model to examine how optimal transit networks should be developed based on changes in input elements of the transit network. Three major inputs-demand, travel speed, and transfer penalty-are chosen for the sensitivity analysis. Different optimal transit networks and their characteristics are generated, and the relationship between inputs and outputs is discussed. Using the sensitivity analysis, three typical transit networks-transferoriented transit, transfer-avoidance transit, and directly-connected transit-are introduced. Optimal types of transit networks are suggested based on transit network situations.
\end{abstract}

\section{Introduction}

It is not easy to design an optimal transit network because of complexity in formulation and optimization. Although current techniques of optimization enable operators to design more efficient transit networks, optimizing whole networks is extremely costly and presents difficulties in implementing changes.

The situation around the transit network changes everyday. Although those changes appear small, after a certain period of time they can become big enough 
to alter the transit network. However, designing a totally new transit network is not easy because of the complexity of the optimization process and the users' ability to adapt to a totally new transit network. Thus, rather than designing a new transit network, in many situations modifying an existing one is a better alternative. In modifying a transit network, it is important to understand the relationship between transit network design inputs and outputs. To produce outputs using different inputs for the transit network, it is necessary to build a model to generate a transit network. In this research, Lee's model (Lee 1998; Lee and Vuchic 2005) is used.

With Lee's model, first basic network inputs are applied. Then, to pursue sensitivity analysis, different inputs are used to compare the outputs, so the relationship between inputs and outputs can be analyzed. Finally, using the results of the sensitivity analysis, three typical types of the transit networks are developed.

\section{The Model for the Transit Network Design}

Much research has been done to improve transit network design. Numerous scholars, including Newell (1979) and Baaj and Mahmassani (1991), have pointed out that traditional mathematical programming has difficulties in generating an optimal transit network due to nonlinearity and nonconvexity of the model, combinatorial explosion, multiobjective nature, and spatial layout of routes. With the improvement of search algorithms and computer technology, important heuristic research has been done (Hasselström 1981; Baaj and Mahmassani 1991; Shih, Mahmassani, and Baaj 1998; Ceder and Israeli 1998; Pattnaik, Mohan, and Tom 1998; Chien, Yang, and Hou 2001). All of these studies are based on the combinatorial search approach.

One key point of the combinatorial approach is efficient generation of sample spaces, which are candidate routes and candidate sets of routes. Depending on the generated sample spaces, the optimality of the results is basically decided, even if an improvement procedure follows. Also, the number of generated candidate routes and candidate sets of routes are critical in this method. If the numbers are too large, then this method becomes close to the all-enumeration method. If they are too small, it is hard to generate good routes and sets of routes for the sample spaces. Thus, this approach tends to rely on the network designer's knowledge to obtain a good simplified sample space. Also, consistency and generalization of the network designer's knowledge are required. Another key point is the flexibility of 
the methodology in respect to handling constraints. Although the combinatorial search approach may yield good results with given fixed inputs, it is not flexible enough to include certain dynamic inputs, particularly those such as variable transit demand.

Lee's model uses the iterative approach to solving the transit network design problem. This approach is flexible enough to deal with dynamic characteristics of transit network design. To execute this methodology, the computer software TRANED (TRAnsit NEtwork Designer) was programmed with C++.

\section{Algorithm of the TRANED}

Unlike auto travel, which increases auto travel time with increased auto travel demand due to congestion, increased transit travel demand decreases transit travel time due to the higher service frequency. However, to have more transit riders under fixed transit demand, circuitous routing is unavoidable. Circuitous routing results from a trade-off relationship between in-vehicle travel time and waiting time in a transit network. The methodology of this research is based on the "concentration of flow" concept, which was introduced and used by Rea (1971) and Hasselström (1981), although they limited its usage to the realization and applications as mentioned.

The iterative approach in this article looks for the minimum total travel time network starting from generating the minimum in-vehicle travel time network. The transit network is gradually improved by increasing in-vehicle travel time while decreasing waiting time. This algorithm consists of three major steps: generation of an initial network, assignment, and network improvement. They are followed by a supporting step, network analysis. These steps are iterated until the optimal transit network is generated, as shown in Figure 1. The generated optimal transit network provides direct connections to major travel flows, while also providing shorter waiting times to minor travel flows by generating circuitous travel paths.

The first step involves generating the initial network with the minimum number of routes using the shortest path algorithm (Dijkstra 1959; Whiting and Hillier 1960; Dantzig 1967). This step provides minimum in-vehicle travel time paths to all origin-destination pairs. For this procedure, the shortest paths for all origin-destination pairs are generated; included paths are then eliminated to avoid unnecessary overlapping paths.

The second step repeats the transit assignment procedure, which concentrates transit travel flow to certain routes. This procedure allows higher frequencies of 


\section{Figure 1. Final Procedure for Transit Network Design for the Basic TRANED Model}

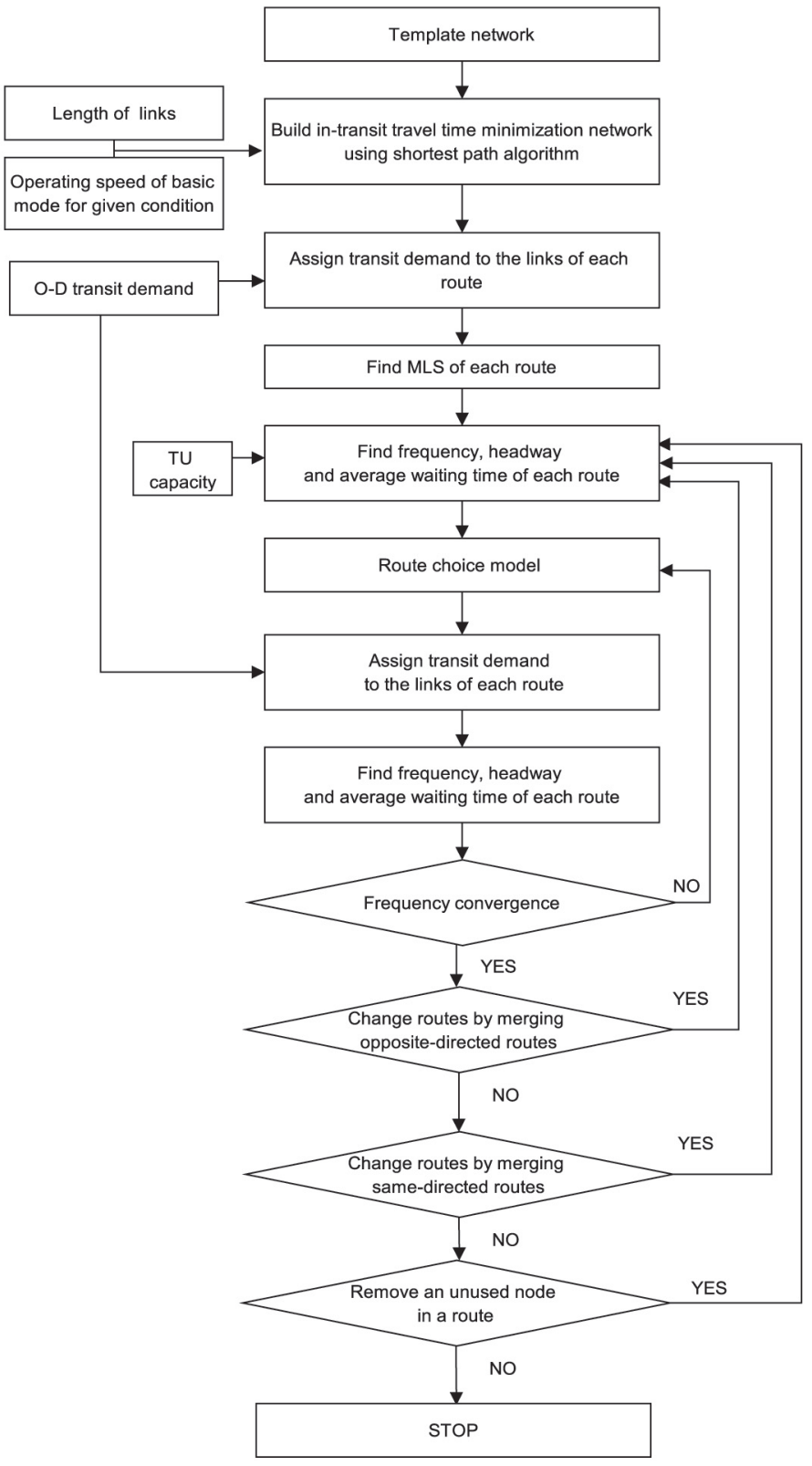


certain routes and shorter total travel time. As a result, less efficient routes are eliminated from the network.

The third step improves the transit network by changing the alignments of routes. After building an initial network and adjusting it to assignment procedure, some alignment changes of certain routes for the improvement of the network should be considered for reducing users' travel times. After stabilizing frequencies of routes in the transit network through repeated assignment procedures, routes are reviewed and alignments are changed where necessary. Less frequent routes require longer waiting times that cause longer travel times so they would be considered first. Since the network consists of selected routes, routes in Baaj and Mahmassani's initial network may need to be split and branches changed in addition to merging routes (1991). However, the procedure in this analysis merges routes and removes unused nodes for network improvements, because the initial network of this study starts from all shortest travel time routes.

There are two cases for merging routes. One involves merging routes that have shared trucks and same-directed branches; the other has shared trucks and opposite-directed branches. If branches of two routes go from the same station of the shared trunk section, it is called same-directed branches. If branches of two routes go from the different stations of the shared trunk section, it is referred to as opposite-directed branches.

Network analysis is the supporting step to generate outputs resulting from the above steps. The outputs of each step, such as number of routes, total travel time, and frequency of routes, are compared to those of the previous step.

The results of this procedure were generated and compared with other research (Mandle 1979; Baaj and Mahmassani 1991) to prove the validation of the methodology (Lee 1998). The results show that transit networks generated by TRANED generally require less travel time for users.

This basic model is simple; however, because of the flexibility of the mathematical programming of the iterative approach, this methodology can add various realistic constraints to the basic model. Additional constraints to those in the basic model are operational and financial limitations, coordination with existing service (intermodal coordination), express service, schedule information for users, and variable transit demand. 


\section{Inputs and Outputs of the Transit Network}

To generate a transit network using Lee's model, input elements for the model required are as follows:

- Template network (basic network with links and nodes);

- Origin-destination travel demand;

- Distance or in-vehicle travel time on each link by mode;

- Transit unit (TU) capacity of given mode;

- Relative weight for waiting time compared to in-vehicle travel time;

- Transfer penalty; and

- Relative weight for transfer time compared to in-vehicle travel time.

For the purpose of analyzing the network generated by TRANED, the following network characteristics are also computed by TRANED in addition to the basic output-network configuration and frequencies of routes:

- Network configuration or route configurations [-];

- Frequencies of routes [vehicle/h];

- Total in-vehicle travel time in the network [person-minutes/h];

- Total waiting time in the network [prs-min/h];

- Total transfer time in the network [prs-min/h];

- Total transfer penalties in the network [prs-min/h];

- Total travel time in the network [prs-min/h];

- Total travel time except in-vehicle travel time [prs-min/h];

- Travel demand without transfer [prs];

- Travel demand requiring transfer [prs];

- Total travel demand [prs];

- Degree of circuity [\%];

- Number of routes [-];

- Total route length in the network $[\mathrm{km}]$;

- Average route length $[\mathrm{km}]$; and

- Total vehicle operational time in the network [veh-min/h]. 
Most of the outputs are self-explanatory, but some require additional explanation. The degree of circuity is the parameter showing the indirectness of travel. There are two types of circuities: physical circuity and time circuity. While physical circuity represents circuity of routes, time circuity represents circuity of travel. The main differences between the two are transfer time and penalty. While physical circuity does not include transfer time and penalty as extra costs, time circuity considers them as extra costs due to the indirectness of a route. Time circuity, used in this study, is the ratio of the extra travel time after boarding a transit vehicle due to the indirectness of routes, possible transfer time, and transfer penalties to the shortest in-vehicle travel time (equation 1). Degree of circuity in the network is the average of an individual user's degree of circuity.

where:

$$
\mathrm{DOC}[\%]=100 \cdot \frac{\Delta t_{i}+t_{t}+p}{\min t_{i}},
$$

$\Delta t_{i} \quad$ represents additional in-vehicle travel time (difference between real in-vehicle travel time and in-vehicle travel time of shortest path)

$t_{i} \quad$ equals transfer time

$p \quad$ represents transfer penalty

$\min t_{i} \quad$ is in-vehicle travel time of shortest path

Total vehicle operational time in the network, which is the accumulation of the vehicle operating time in the network, is calculated as follows. (The 2 in the equation means two-directional service, which is conventional in most transit service.) where:

$$
\text { Total vehicle operational time }(\mathrm{TOT})=2 \sum_{k} f_{k} \cdot l_{k},
$$

$k \quad$ is the route number

$f$ equals frequency

$l$ is the length of route in minutes or operating time for one direction 


\section{Basic Network}

The network and other inputs to be used in this example come from Rea's study (1971), although the demand and the length of links are modified to provide more realistic results. The configuration of the network and basic inputs are shown in the Figure 2.

As other input elements for the model, TU capacity, transfer penalty, and relative weight for waiting time and transfer time must be defined. For TU capacity, 60 spaces, used in Rea's paper, is also applied. For simplicity, no transfer penalty is applied for the basic case. That means there are no additional fares, additional access times, and other qualitative inconveniences related to transfers. As a relative weight of the waiting time to in-vehicle travel time, the ratio of 1 is used, which means the values of waiting time and in-vehicle travel time are the same for the simplicity as well.

\section{Figure 2. Inputs for the Basic Case}

\section{(a) Template network}

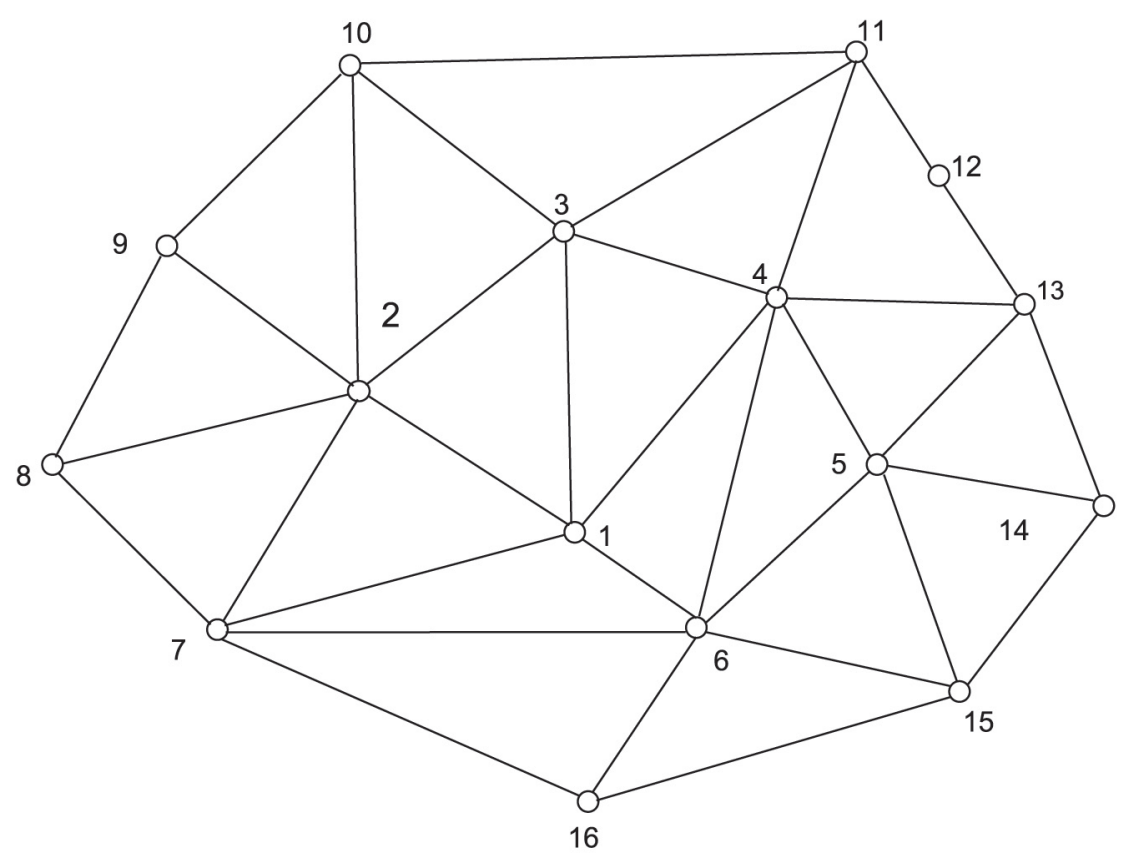




\section{(b) Travel time of each link}

(min)
\begin{tabular}{|l|l|l|l|l|l|l|l|l|l|l|l|l|l|l|l|l|}
\hline Node & $\# 1$ & $\# 2$ & $\# 3$ & $\# 4$ & $\# 5$ & $\# 6$ & $\# 7$ & $\# 8$ & $\# 9$ & $\# 10$ & $\# 11$ & $\# 12$ & $\# 13$ & $\# 14$ & $\# 15$ & $\# 16$ \\
\hline$\# 1$ & 0 & 2.36 & 2.78 & 2.36 & 0 & 2.23 & 2.38 & 0 & 0 & 0 & 0 & 0 & 0 & 0 & 0 & 0 \\
\hline$\# 2$ & 2.36 & 0 & 2.00 & 0 & 0 & 0 & 2.84 & 2.48 & 2.00 & 2.22 & 0 & 0 & 0 & 0 & 0 & 0 \\
\hline$\# 3$ & 2.78 & 2.00 & 0 & 2.00 & 0 & 0 & 0 & 0 & 0 & 2.00 & 2.48 & 0 & 0 & 0 & 0 & 0 \\
\hline$\# 4$ & 2.36 & 0 & 2.00 & 0 & 2.00 & 2.84 & 0 & 0 & 0 & 0 & 2.28 & 0 & 2.78 & 0 & 0 & 0 \\
\hline$\# 5$ & 0 & 0 & 0 & 2.00 & 0 & 2.00 & 0 & 0 & 0 & 0 & 0 & 0 & 2.36 & 2.98 & 2.28 & 0 \\
\hline$\# 6$ & 1.12 & 0 & 0 & 2.84 & 2.00 & 0 & 3.34 & 0 & 0 & 0 & 0 & 0 & 0 & 0 & 2.48 & 2.00 \\
\hline$\# 7$ & 2.48 & 2.84 & 0 & 0 & 0 & 3.34 & 0 & 2.36 & 0 & 0 & 0 & 0 & 0 & 0 & 0 & 2.78 \\
\hline$\# 8$ & 0 & 2.48 & 0 & 0 & 0 & 0 & 2.36 & 0 & 1.96 & 0 & 0 & 0 & 0 & 0 & 0 & 0 \\
\hline$\# 9$ & 0 & 2.00 & 0 & 0 & 0 & 0 & 0 & 2.28 & 0 & 2.00 & 0 & 0 & 0 & 0 & 0 & 0 \\
\hline$\# 10$ & 0 & 2.22 & 2.00 & 0 & 0 & 0 & 0 & 0 & 2.00 & 0 & 3.62 & 0 & 0 & 0 & 0 & 0 \\
\hline$\# 11$ & 0 & 0 & 2.48 & 2.28 & 0 & 0 & 0 & 0 & 0 & 3.62 & 0 & 1.12 & 0 & 0 & 0 & 0 \\
\hline$\# 12$ & 0 & 0 & 0 & 0 & 0 & 0 & 0 & 0 & 0 & 0 & 1.12 & 0 & 1.12 & 0 & 0 & 0 \\
\hline$\# 13$ & 0 & 0 & 0 & 2.78 & 2.36 & 0 & 0 & 0 & 0 & 0 & 0 & 1.12 & 0 & 2.98 & 0 & 0 \\
\hline$\# 14$ & 0 & 0 & 0 & 0 & 2.98 & 0 & 0 & 0 & 0 & 0 & 0 & 0 & 2.98 & 0 & 2.48 & 0 \\
\hline$\# 15$ & 0 & 0 & 0 & 0 & 2.28 & 2.48 & 0 & 0 & 0 & 0 & 0 & 0 & 0 & 2.48 & 0 & 3.38 \\
\hline$\# 16$ & 0 & 0 & 0 & 0 & 0 & 2.00 & 2.78 & 0 & 0 & 0 & 0 & 0 & 0 & 0 & 3.38 & 0 \\
\hline
\end{tabular}

\section{(c) Origin-destination demand}

\begin{tabular}{|l|l|l|l|l|l|l|l|l|l|l|l|l|l|l|l|l|}
\hline Node & $\# 1$ & $\# 2$ & $\# 3$ & $\# 4$ & $\# 5$ & $\# 6$ & $\# 7$ & $\# ~ 8$ & $\# 9$ & $\# 10$ & $\# 11$ & $\# 12$ & $\# 13$ & $\# 14$ & $\# 15$ & $\# 16$ \\
\hline$\# 1$ & 0 & 10 & 10 & 10 & 10 & 10 & 0 & 0 & 0 & 0 & 0 & 0 & 0 & 0 & 0 & 0 \\
\hline$\# 2$ & 60 & 0 & 40 & 60 & 40 & 40 & 20 & 20 & 20 & 20 & 20 & 20 & 20 & 20 & 20 & 20 \\
\hline$\# 3$ & 60 & 40 & 0 & 60 & 40 & 40 & 20 & 20 & 20 & 20 & 20 & 20 & 20 & 20 & 20 & 20 \\
\hline$\# 4$ & 10 & 10 & 10 & 0 & 10 & 10 & 0 & 0 & 0 & 0 & 0 & 0 & 0 & 0 & 0 & 0 \\
\hline$\# 5$ & 60 & 40 & 40 & 6 & 0 & 40 & 20 & 20 & 20 & 20 & 20 & 20 & 20 & 20 & 20 & 20 \\
\hline$\# 6$ & 60 & 40 & 40 & 60 & 40 & 0 & 20 & 20 & 20 & 20 & 20 & 20 & 20 & 20 & 20 & 20 \\
\hline$\# 7$ & 80 & 20 & 20 & 80 & 20 & 20 & 0 & 10 & 10 & 10 & 10 & 10 & 10 & 10 & 10 & 10 \\
\hline$\# 8$ & 80 & 20 & 20 & 80 & 20 & 20 & 10 & 0 & 10 & 10 & 10 & 10 & 10 & 10 & 10 & 10 \\
\hline$\# 9$ & 80 & 20 & 20 & 80 & 20 & 20 & 10 & 10 & 0 & 10 & 10 & 10 & 10 & 10 & 10 & 10 \\
\hline$\# 10$ & 80 & 20 & 20 & 80 & 20 & 20 & 10 & 10 & 10 & 0 & 10 & 10 & 10 & 10 & 10 & 10 \\
\hline$\# 11$ & 80 & 20 & 20 & 80 & 20 & 20 & 10 & 10 & 10 & 10 & 0 & 10 & 10 & 10 & 10 & 10 \\
\hline$\# 12$ & 80 & 20 & 20 & 80 & 20 & 20 & 10 & 10 & 10 & 10 & 10 & 0 & 10 & 10 & 10 & 10 \\
\hline$\# 13$ & 80 & 20 & 20 & 80 & 20 & 20 & 10 & 10 & 10 & 10 & 10 & 10 & 0 & 10 & 10 & 10 \\
\hline$\# 14$ & 80 & 20 & 20 & 80 & 20 & 20 & 10 & 10 & 10 & 10 & 10 & 10 & 10 & 0 & 10 & 10 \\
\hline$\# 15$ & 80 & 20 & 20 & 80 & 20 & 20 & 10 & 10 & 10 & 10 & 10 & 10 & 10 & 10 & 0 & 10 \\
\hline$\# 16$ & 80 & 20 & 20 & 80 & 20 & 20 & 10 & 10 & 10 & 10 & 10 & 10 & 10 & 10 & 10 & 0 \\
\hline
\end{tabular}


The TRANED solution algorithm changes the optimal transit network through iterations (Table 1).

\section{Sensitivity Analysis}

Three major input elements are used in the sensitivity analysis: demand, travel times on the links, and transfer penalties. The results depend not only on one input component but also on other input components, which means the choice of values for the basic inputs is very important. For example, depending on the size of demand, the sensitivity of another input, such as transfer penalty, can vary greatly. Depending on other given inputs, the output may be more or less sensitive for a specific input.

Because of different inputs, generated transit networks and their characteristics are fairly different. Generated networks cannot be compared directly, so their characteristics should be compared. Among outputs introduced previously, total travel time (TTT), number of routes (NOR), total route length (TRL), average route length (ARL), degree of circuity (DOC), ratio of direct demand without transfer to total transfer (DWOT), total vehicle operating length (TVOT), and some other new outputs are chosen for the comparison.

Unlike analyzing a single network, sensitivity analysis of different networks with different inputs requires adjustment of outputs for the comparison. While some outputs can be directly compared, direct comparison does not mean anything when outputs are directly dependent on inputs. For example, when transit operating speed decreases, in-vehicle travel time is surely increased regardless of the transit network configuration. Also, when transit demand increases, total travel time in the network is increased because of the increased number of passengers. In these cases, outputs should be adjusted for comparing transit networks.

To adjust direct impact of inputs to compare generated network configurations, several versions of outputs are introduced for the sensitivity analysis. These versions are created by assuming that basic inputs are applied to the transit networks generated with different inputs. For example, with all other inputs the same, doubled transit operating speed creates different optimal network configuration compared to that with the basic operating speed. Also, the network with the doubled operating speed requires about half of the total travel time due to reduced in-vehicle travel time just by itself. So, to find out the difference between two network configurations, the same operating speed (basic speed) should be applied to two differently generated networks and the outputs should be compared. 
Table 1. Outputs of the Basic Case

\section{(a) Changes in Routes of the Basic Case}

\begin{tabular}{|l|l|l|}
\hline Iteration & Procedure & Routes \\
\hline Initial & & $\# 1$ to \#28 \\
\hline 1 & Assignment & $\# 1, \# 2, \# 3, \# 5, \# 6, \# 7, \# 8, \# 9, \# 11, \# 12, \# 13, \# 14, \# 15, \# 16, \# 19, \# 23$ \\
\hline 2 & Assignment & $\# 1, \# 2, \# 3, \# 6, \# 7, \# 8, \# 11, \# 12, \# 13, \# 14, \# 16$ \\
\hline 3 & Assignment & $\# 1, \# 3, \# 6, \# 7, \# 8, \# 11, \# 12, \# 14, \# 16$ \\
\hline 4 & Assignment & $\# 1, \# 3, \# 6, \# 7, \# 8, \# 11, \# 12, \# 14$ \\
\hline 5 & Assignment & $\# 1, \# 3, \# 6, \# 7, \# 8, \# 11, \# 12, \# 14$ \\
\hline 6 & Assignment & $\# 1, \# 3, \# 6, \# 7, \# 8, \# 11, \# 12$ \\
\hline 7 & Assignment & $\# 3, \# 6, \# 7, \# 8, \# 11, \# 12$ \\
\hline 8 & Assignment & $\# 3, \# 6, \# 7, \# 8, \# 11, \# 12$ \\
\hline 9 & Assignment & $\# 3, \# 6, \# 7, \# 8, \# 11, \# 12$ \\
\hline 10 & Assignment & $\# 3, \# 6, \# 7, \# 8, \# 11, \# 12$ \\
\hline 11 & Merging & $\# 3(7-1-4-11-12-13), \# 7, \# 8, \# 11, \# 12$ \\
\hline 12 & Assignment & $\# 3(7-1-4-11-12-13), \# 7, \# 8, \# 11, \# 12$ \\
\hline 13 & Assignment & $\# 3(7-1-4-11-12-13), \# 7, \# 8, \# 11, \# 12$ \\
\hline 14 & Assignment & $\# 3(7-1-4-11-12-13), \# 7, \# 8, \# 11, \# 12$ \\
\hline 15 & Merging & $\# 3(7-1-4-11-12-13), \# 7(10-9-2-1-6-16-15-14), \# 11, \# 12$ \\
\hline 16 & Assignment & $\# 3(7-1-4-11-12-13), \# 7(10-9-2-1-6-16-15-14), \# 11, \# 12$ \\
\hline 17 & Assignment & $\# 3(7-1-4-11-12-13), \# 7(10-9-2-1-6-16-15-14), \# 11, \# 12$ \\
\hline
\end{tabular}

\section{(b) Network Characteristics of the Basic Case}

\begin{tabular}{|l|l|l|r|r|r|r|r|r|r|r|}
\hline $\begin{array}{l}\text { Iteration } \\
\text { Number }\end{array}$ & $\begin{array}{l}\text { In-vehicle } \\
\text { Travel } \\
\text { (p.-min) }\end{array}$ & $\begin{array}{l}\text { Waiting } \\
\text { Time } \\
\text { (p.-min) }\end{array}$ & $\begin{array}{l}\text { Transfer } \\
\text { Time } \\
\text { (p.-min) }\end{array}$ & $\begin{array}{l}\text { Total } \\
\text { Travel } \\
\text { Time } \\
\text { (p.-min) }\end{array}$ & $\begin{array}{l}\text { Direct } \\
\text { Demand } \\
\text { (prs/h) }\end{array}$ & $\begin{array}{l}\text { Transfer } \\
\text { Required } \\
\text { Demand } \\
\text { (prs/h) }\end{array}$ & $\begin{array}{l}\text { No. of } \\
\text { Routes }\end{array}$ & $\begin{array}{l}\text { Degree } \\
\text { of } \\
\text { Circuity } \\
(\%)\end{array}$ & $\begin{array}{l}\text { Length } \\
\text { of } \\
\text { Network } \\
\text { (km) }\end{array}$ & $\begin{array}{l}\text { Total } \\
\text { Vehicle } \\
\text { Operating } \\
\text { Time } \\
\text { (veh-min/h) }\end{array}$ \\
\hline Initial & 22577.5 & 105421.0 & 0.0 & 127998.5 & 5160 & 0 & 28 & 0.0 & 97.6 & 671.0 \\
\hline $1^{*}$ & 24805.4 & 49649.9 & 5823.3 & 80278.6 & 4180 & 980 & 16 & 35.7 & 58.0 & 789.7 \\
\hline $2^{*}$ & 25794.0 & 31214.2 & 8296.3 & 65304.5 & 3250 & 1910 & 11 & 51.0 & 41.4 & 884.3 \\
\hline $3^{*}$ & 25727.6 & 26906.2 & 8122.5 & 60756.3 & 3250 & 1910 & 9 & 49.9 & 35.4 & 825.5 \\
\hline $4^{*}$ & 25777.6 & 23919.8 & 7382.4 & 57079.8 & 3150 & 2010 & 8 & 46.9 & 31.9 & 845.2 \\
\hline $5^{*}$ & 26101.6 & 25261.9 & 7483.1 & 58846.6 & 3030 & 2130 & 8 & 48.6 & 31.9 & 853.3 \\
\hline $6^{*}$ & 26306.0 & 21824.6 & 7820.5 & 55951.1 & 2920 & 2240 & 7 & 51.2 & 28.9 & 861.2 \\
\hline $7^{*}$ & 26583.2 & 18406.0 & 8308.3 & 53297.5 & 2720 & 2440 & 6 & 54.4 & 25.1 & 864.2 \\
\hline $8^{*}$ & 26386.4 & 17973.2 & 8195.4 & 52555.0 & 2720 & 2440 & 6 & 53.2 & 25.1 & 894.6 \\
\hline $9^{*}$ & 26386.4 & 17927.1 & 8177.5 & 52491.0 & 2720 & 2440 & 6 & 53.1 & 25.1 & 898.1 \\
\hline $10^{*}$ & 26386.4 & 17973.2 & 8195.4 & 52555.0 & 2720 & 2440 & 6 & 53.2 & 25.1 & 894.6 \\
\hline $11^{* * *}$ & 26943.2 & 16514.6 & 7679.1 & 51136.9 & 2720 & 2440 & 5 & 53.3 & 21.9 & 898.7 \\
\hline $12^{*}$ & 26943.2 & 16400.9 & 7661.5 & 51005.6 & 2720 & 2440 & 5 & 53.3 & 21.9 & 882.2 \\
\hline $13^{*}$ & 26943.2 & 16447.1 & 7679.3 & 51069.6 & 2720 & 2440 & 5 & 53.3 & 21.9 & 878.7 \\
\hline $14^{*}$ & 26943.2 & 16400.9 & 7661.5 & 51005.6 & 2720 & 2440 & 5 & 53.3 & 21.9 & 882.2 \\
\hline $15^{* * *}$ & 29623.2 & 12591.4 & 6163.2 & 48377.8 & 2840 & 2320 & 4 & 58.5 & 20.5 & 1081.8 \\
\hline $16^{*}$ & 29462.4 & 13034.7 & 6352.8 & 48849.9 & 2840 & 2320 & 4 & 58.6 & 20.5 & 1012.1 \\
\hline $17^{*}$ & 29429.6 & 13087.6 & 6366.3 & 48883.5 & 2840 & 2320 & 4 & 58.5 & 20.5 & 1002.3 \\
\hline
\end{tabular}


Also, when different sizes of demand are applied for the sensitivity analysis, travel time per person or per trip (TTT/trip) is used as adjusted values for the same reason.

The adjusted versions of outputs, which are adjusted total travel time (ATTT), adjusted total vehicle operating time (ATVOT), etc, will be explained in detail with sensitivity analysis.

\section{Changes in Demand Level}

Different demand levels, which are the basic demand and the following multiples of $0.5,1.5,2,2.5$, and 3 , are applied and their results are compared, while other inputs remain the same.

Figure 3 shows the basic relationships between different sizes of demand and the outputs. As expected, in Figure 3(a), total travel time in the network (TTT) increases with the increased number of passengers, but the adjusted total travel time-I (ATTT-I) in the network decreases. As mentioned previously, the adjusted total travel time-l is defined as the value, which assumes the same demand level of the basic case but the same frequencies of the network with the different demand. As shown in the figure, the two curves cross at the same point where demand level is 100 percent, which is the basic case. A decreasing adjusted total travel time-I curve with increased demand shows increased efficiency of the transit system for users. The in-vehicle travel time/trip (IVT/trip) and other than in-vehicle travel time (waiting time and transfer time)/trip (OIVT/trip) are plotted with the secondary $\mathrm{Y}$-axis. These curves show that with increased demand, not only waiting time (WT) and transfer time (TT) decrease, which is rational and surely expected, but also in-vehicle travel time (IVT) decreases. Because both components of travel time decrease, adjusted total travel time-I decreases with increased demand. The adjusted total travel time curve-II (ATTT-II) in the figure, which is plotted with the primary $\mathrm{Y}$-axis, represents the total travel times estimated with basic demand but applied to networks generated by a range of demand. While the adjusted total travel time-I uses the frequencies of given demand, the adjusted total travel timeII uses the adjusted frequency based on the basic demand. Since the networks are generated to minimize the total travel time for each demand level, the basic case (network) with the basic demand provides the least total travel time of all cases (networks). This shows the consistency of the results generated by TRANED.

In Figure 3(b), the percentage of direct demand (DWOT), degree of circuity (DOC), and the number of routes (NOR) are plotted. While the curve for the number of 
routes uses the secondary $\mathrm{Y}$-axis, the other two curves use the primary $\mathrm{Y}$-axis. As shown, the number of routes increases with increased demand. Consequently, demand for direct trips also increases while the degree of circuity decreases with increased demand level.

In Figure 3(c), the total route length in the network (TRL), average route length (ARL), total vehicle operating time (TVOT), and adjusted total vehicle operating time (ATVOT) are analyzed. The first two results are plotted with the primary $\mathrm{Y}$-axis and the last two are plotted with the secondary $\mathrm{Y}$-axis. With the increased number of routes, the total length of network increases, while the average length of route does not decrease much. Total vehicle operating time increases with increased length of the network, and that is as expected to serve more passengers. However, its adjusted version shows the reverse result. Using the demand of the basic case, the networks generated by using increased demand actually require shorter total vehicle operating time.

An interesting and important result is analyzed in Figure 3(d). Two curves, average travel time per passenger (TTT/trip) and average vehicle operating time per passenger (TVOT/trip), are plotted with the primary and secondary $\mathrm{Y}$-axes. It was found that not only average travel time per passenger, but also average vehicle operating time per passenger decrease with increased demand level. This shows that increased transit demand offers a more efficient transit network for both users and operators.

Larger demand level results in higher frequencies, and reduces the necessity of circuitous routing and transferring. Consequently, the number of routes (NOR) increases, demand without transfer (DWOT) increases, directness of travel increases, and average total travel time per passenger and vehicle operating length per passenger decrease. From the indications above, in general, overall efficiency of the network increases as demand increases, and this verifies the commonly held assumption that the marginal cost of transit does decrease with increasing ridership.

\section{Changes in Travel Speed}

This section addresses the relationship between travel times on the links (or operating speed) and the network characteristics generated with the otherwise same inputs. To reflect different operating speeds, different travel times on the links ( 0.5 , $0.75,1,1.25,1.75$, and 2 times the basic in-vehicle travel times) are applied to the model while keeping all other inputs as before. 
Figure 3(a). Analysis with Different Demand Levels

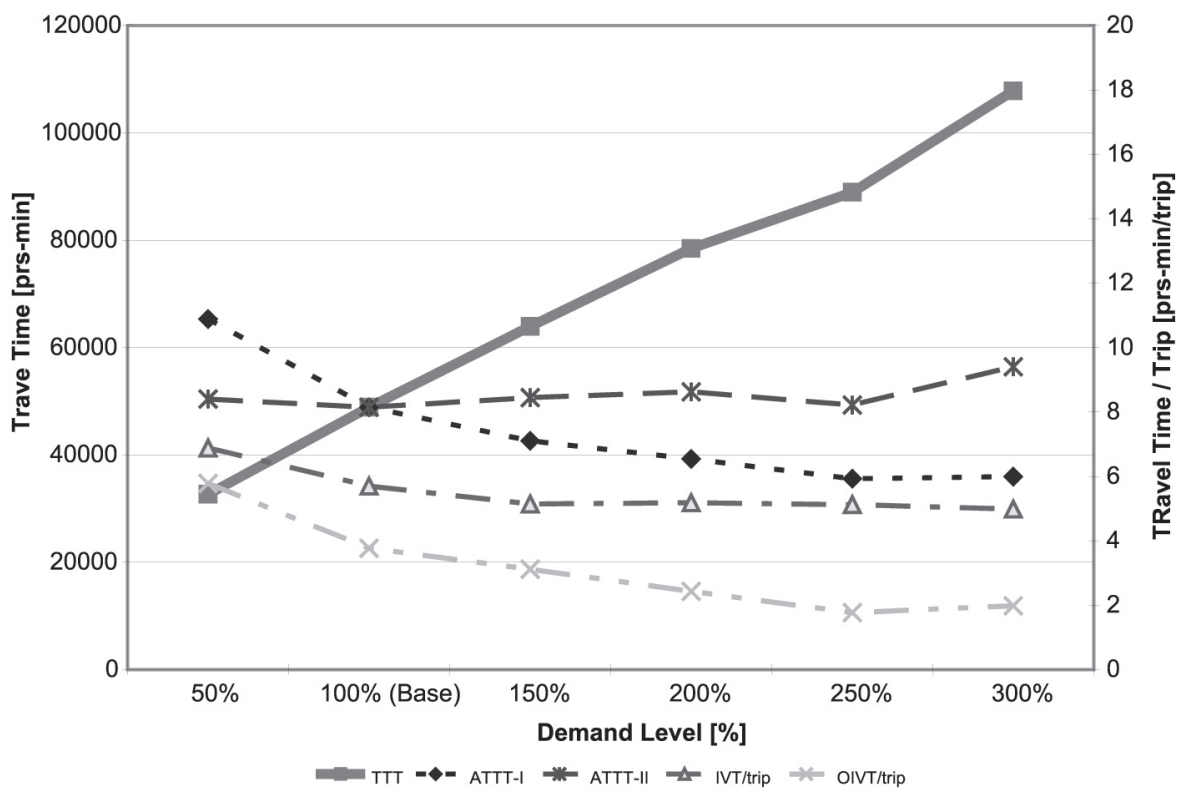

Figure 3(b)

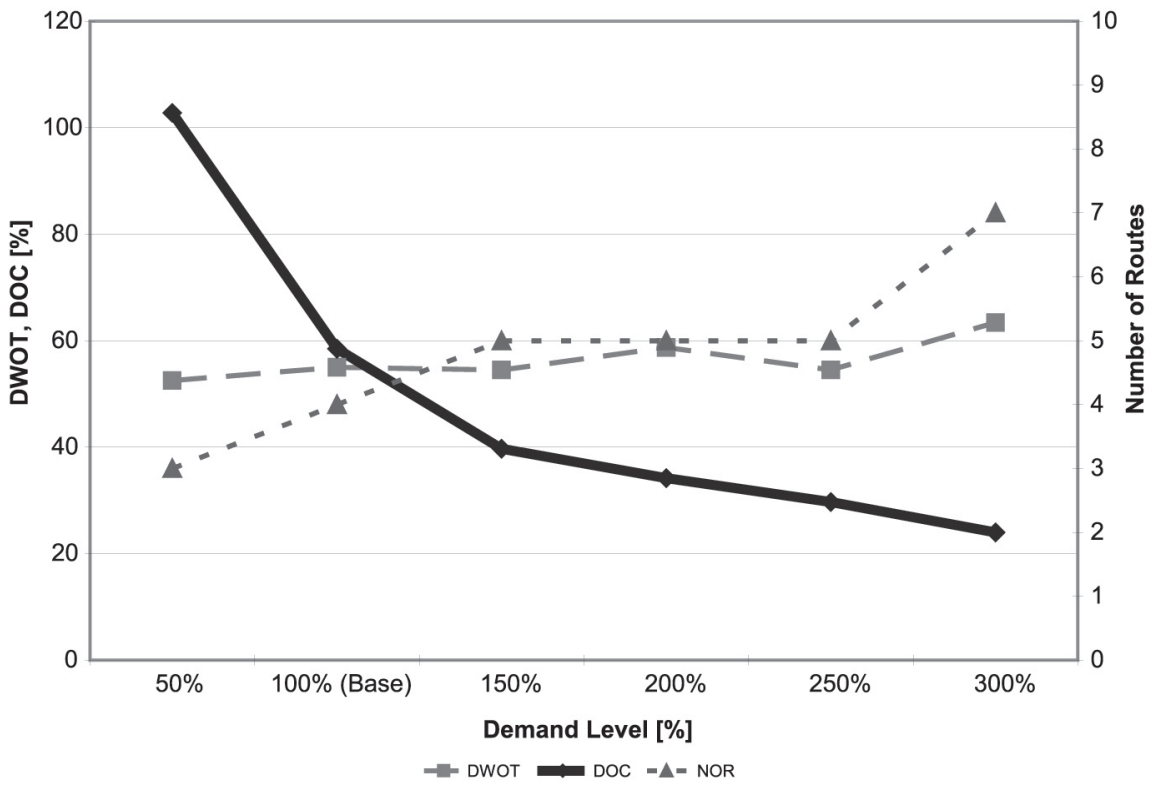


Figure 3(c)

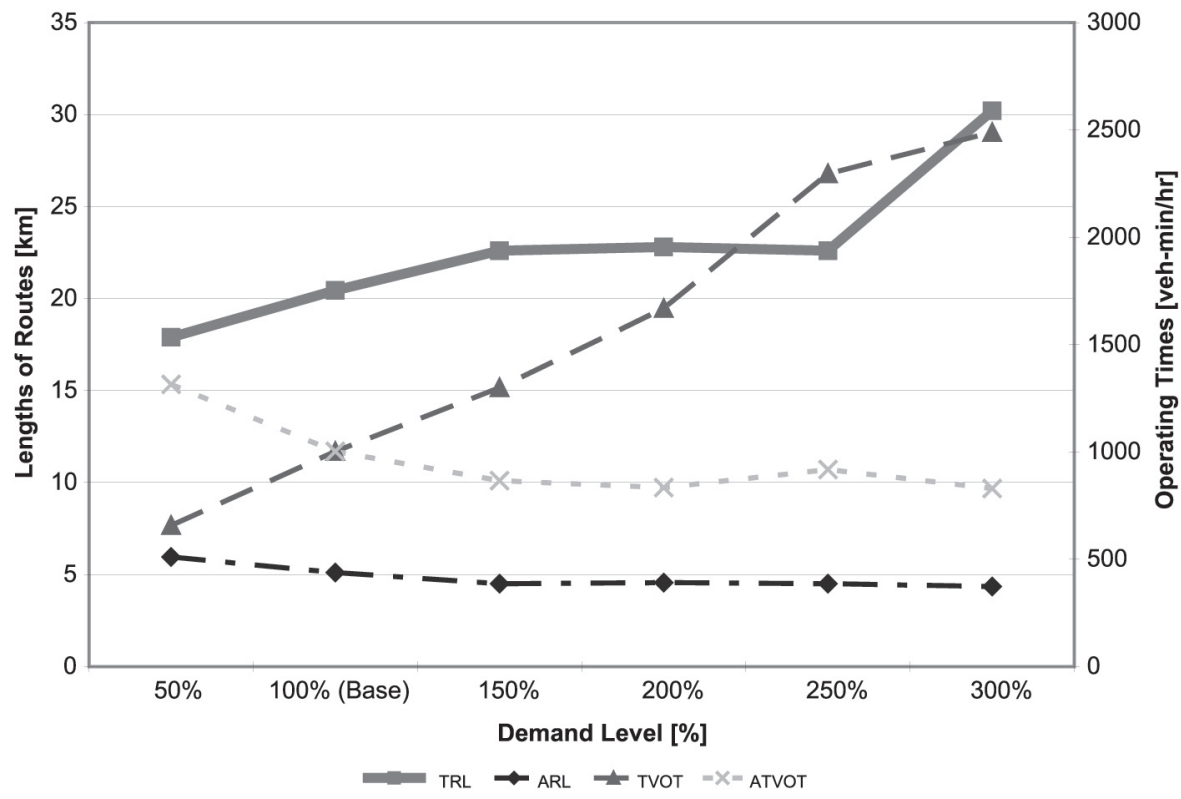

Figure 3(d)

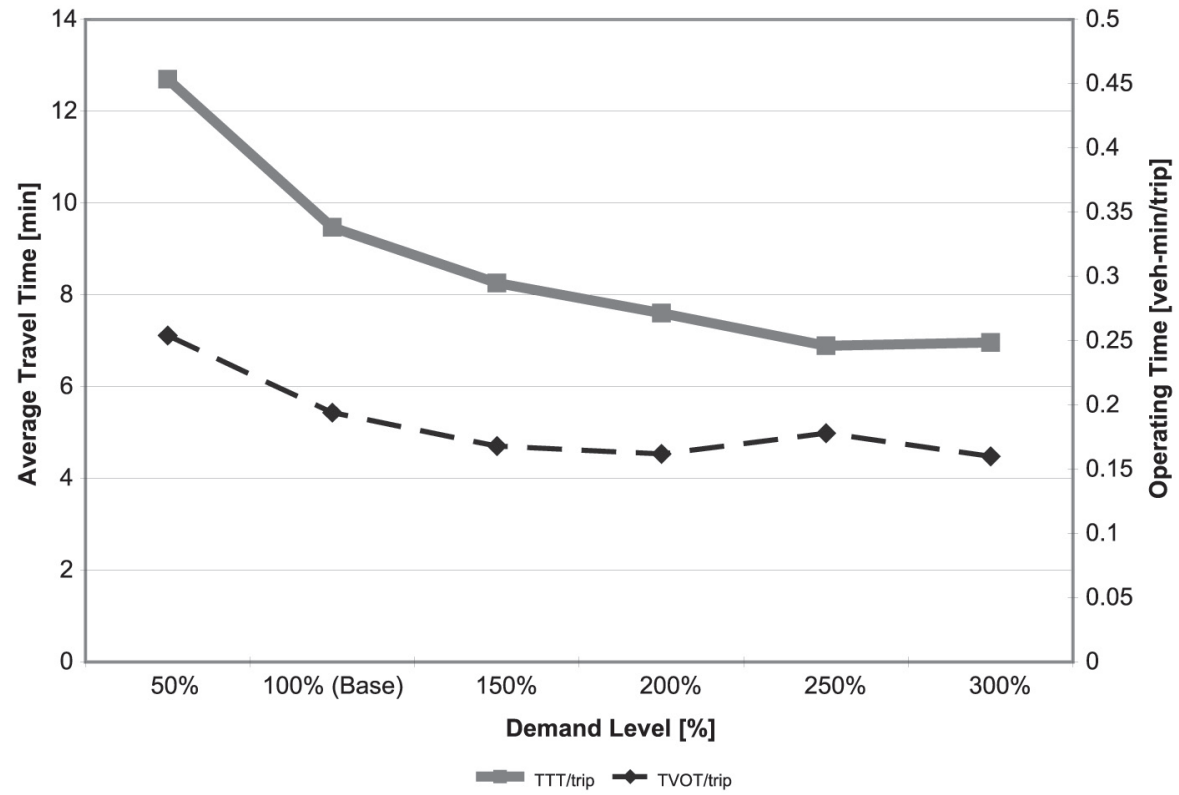


Figure 4 illustrates the basic relationships between speeds and outputs. As shown in Figure 4(a), the curve for the total travel time in the networks (TTT) obviously increases with lower operating speed and longer in-vehicle travel times on the links, as expected. As another characteristic for the network analysis, the curve for the adjusted total travel time (ATTT) is plotted in Figure 4(a), which is the total travel time using the basic operating speed with the networks generated based on the other operating speeds. Since only the network of the basic case is generated with basic in-vehicle-travel time, and the other networks are generated with other in-vehicle travel times, as plotted, the basic case has the minimum adjusted total travel time. This result shows that the networks generated with different in-vehicle travel times and their results are reliable and consistent.

Total travel time outputs, total travel time, and adjusted total travel time are plotted on the primary $\mathrm{Y}$-axis. The ratio of in-vehicle travel time to total travel time (IVT/TTT) and its adjusted version (AIVT/TTT) are plotted on the secondary Y-axis.

While the ratio of in-vehicle travel time to total travel time (IVT/TTT) increases due to increased in-vehicle travel times on the links, the ratio of adjusted in-vehicle travel time to adjusted total travel time (AIVT/TTT) decreases because of the effort to minimize in-vehicle travel time by the network generation procedure.

In Figure 4(b), the percentage of demand without transfer (DWOT) and the degree of circuity $(D O C)$ are plotted on the primary $Y$-axis, while the number of routes $(N O R)$ is plotted on the secondary $Y$-axis. Because of increased in-vehicle travel times on the links, the network provides less circuitous routing and more routes, which require less in-vehicle travel time. As expected, demand without transfer increases and the degree of circuity decreases.

Figure $4(\mathrm{c})$ shows the total route length in the network (TRL) and the average route length $(A R L)$ plotted against in-vehicle travel times. As discussed for Figure $4(b)$, to avoid longer in-vehicle travel time, more direct networks are generated with decreased operating speed. Consequently, the average route length decreases. However, because of the increased number of routes, the length of network increases moderately with increased in-vehicle travel times of the network.

The two curves in Figure 4(d) are total vehicle operating time (TVOT) plotted with the primary $\mathrm{Y}$-axis, of which the unit is vehicle- $\mathrm{min} / \mathrm{h}$ and total vehicle operating distance (TVOD) plotted with the secondary $Y$-axis, of which the unit is vehicle$\mathrm{km} / \mathrm{h}$, both plotted against in-vehicle travel times. While total vehicle operating 
Figure 4(a). Analysis with Changed In-Vehicle Travel Time or Operating Speed

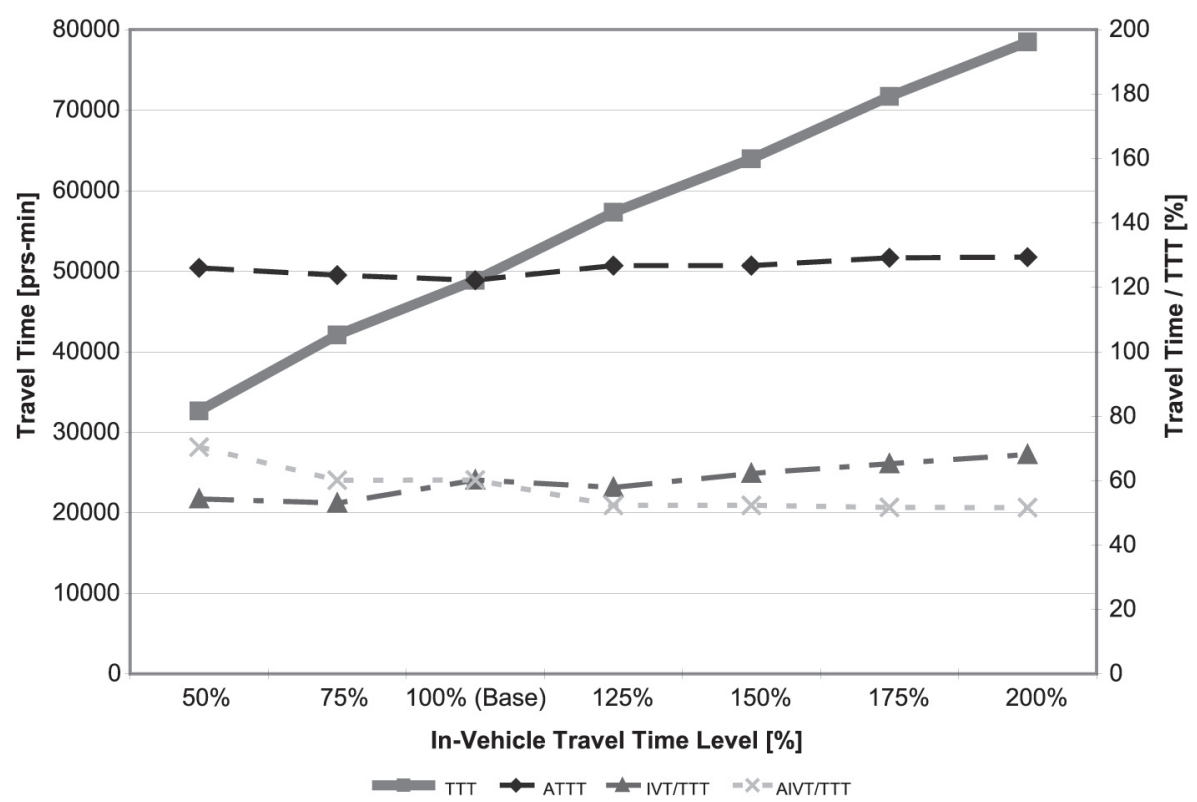

Figure 4(b)

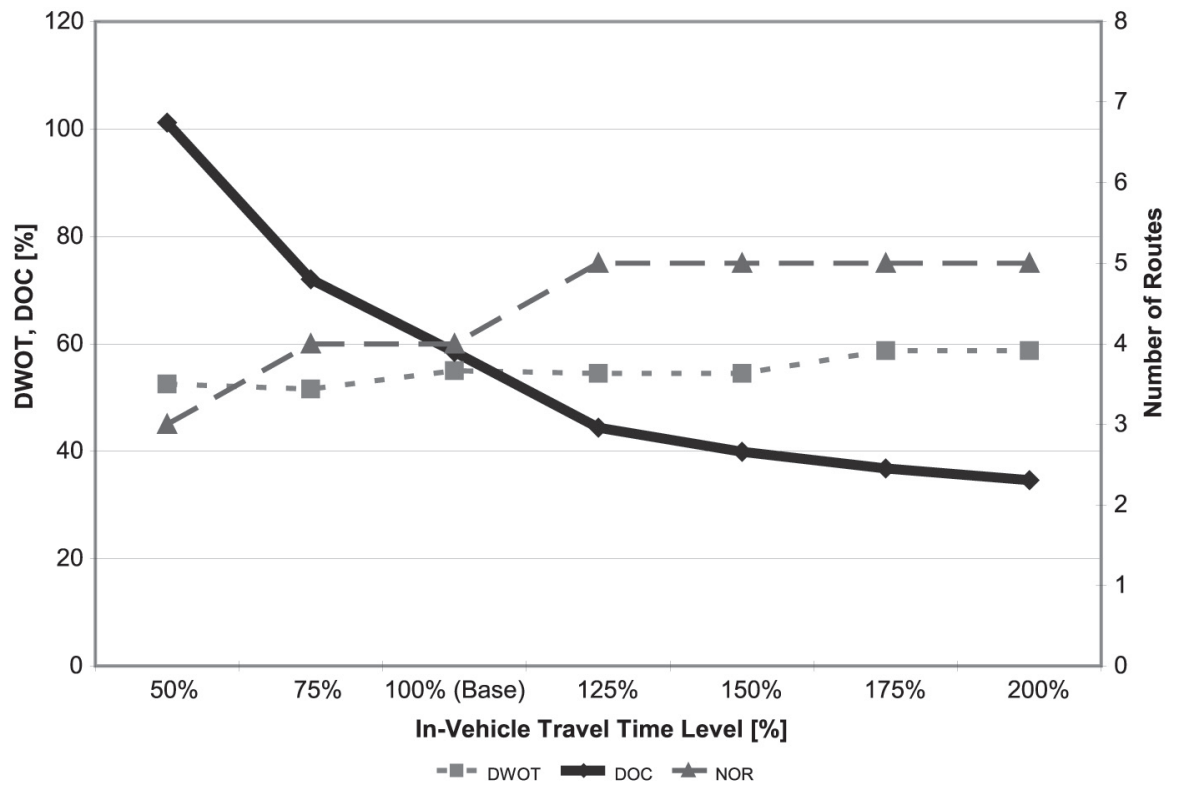


Journal of Public Transportation, Vol. 9, No. 1, 2006

Figure 4(c)

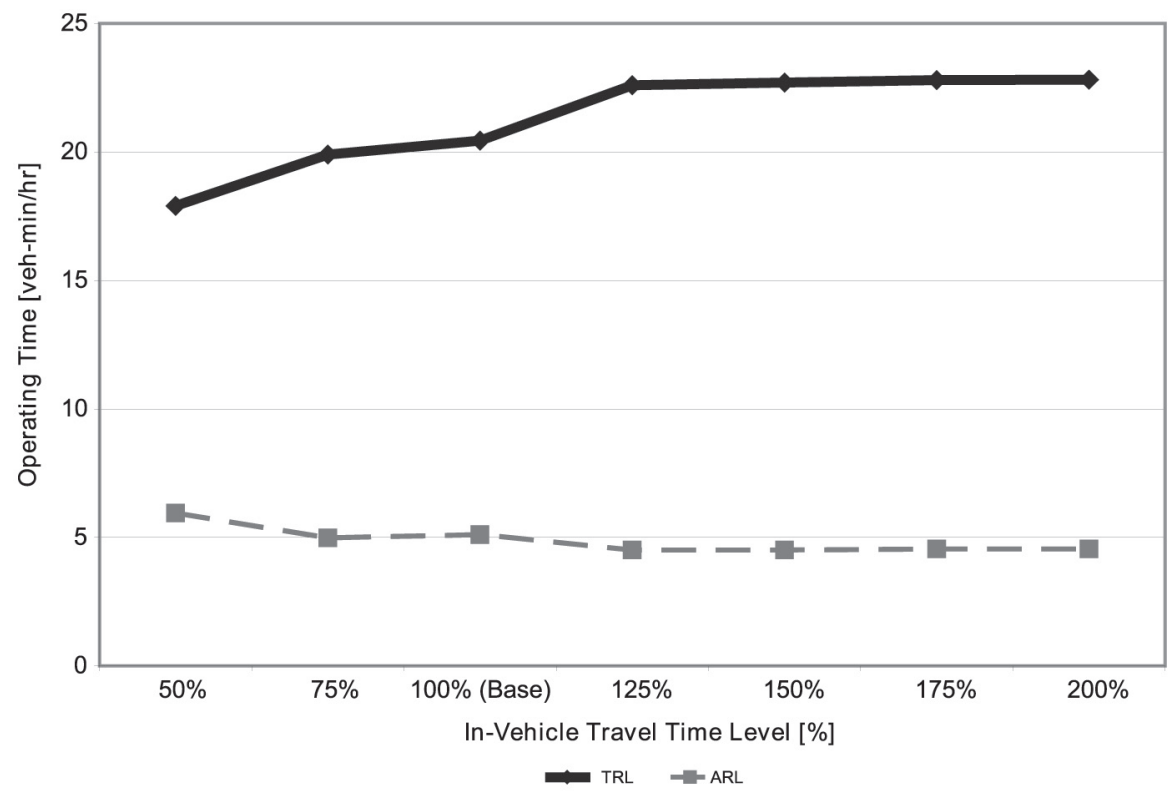

Figure 4(d)

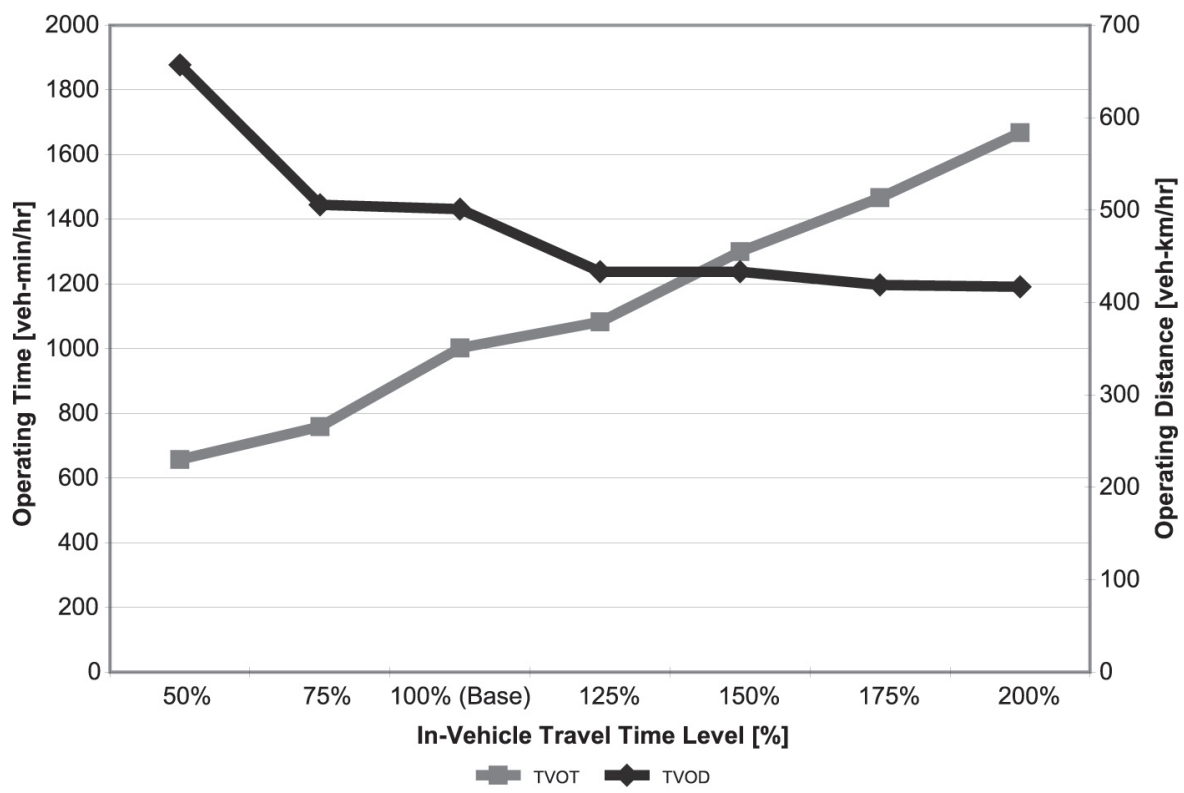


time increases due to increased link costs (in-vehicle time), because of less circuitous routing, total vehicle operating length decreases.

When in-vehicle travel time increases due to the slower speed or congestion, for users' travel time minimization, more routes with shorter length should be formed to provide more direct service.

\section{Changes in Transfer Penalties (Lower Demand Case)}

Depending on other given inputs, the output may be more or less sensitive for a specific input. Especially when the demand size is different, the results of the sensitivity analysis for the transfer penalties can vary greatly. For the sensitivity analysis for the transfer penalties, two cases-lower demand and higher demand-are discussed.

In this section, in addition to waiting time at the transfer station, additional transfer penalties are applied. These penalties can account for an additional fare, access time to a transfer station, and the inconvenience involved in transferring. They are given as equivalent minutes (10, 20, and 30 minutes) of in-vehicle travel time.

In Figure 5(a), total travel time (TTT) and its adjusted version (ATTT) are plotted with the primary $\mathrm{Y}$-axis. While the total travel time includes transfer penalties in addition to in-vehicle travel time (IVT), waiting time (WT) and transfer time $(T T)$, the adjusted version does not include transfer penalties, which means that it represents travel time only. Total travel time (TTT) increases with increased transfer penalty, except for the transfer penalty of 30 minutes, which is an inconsistent result, but within a range of acceptable error. The adjusted total travel time (ATTT) also increases with increased transfer penalty. These two curves show that not only increased transfer penalty, but also that actual travel time components cause total travel time increases. This can be explained by the fact that when the transfer penalty increases significantly, transit networks are inefficiently generated to avoid large transfer penalties.

The ratio of in-vehicle travel time to total travel time (IVT/TTT) and its adjusted version (IVT/ATTT), which does not include transfer penalty in total travel time, are plotted in Figure 5(a) against the secondary $\mathrm{Y}$-axis. With increased transfer penalty, the portion of in-vehicle travel time in the network increases. That is due to circuitous and longer routing to avoid transfers, and will be explained in detail in below.

Network characteristics are plotted in Figure 5(b). To avoid transfers and their penalties, transit networks are generated using fewer but more circuitous routes. 
Thus, both types of degree of circuity, with (DOC) and without (ADOC) taking transfer penalty into account, increase with increased transfer penalty as plotted on the primary $\mathrm{Y}$-axis. The number of routes $(N O R)$ as plotted against secondary $Y$-axis decreases with increased penalty.

Direct-trip demand without transfers (DWOT) increases with increased transfer penalty, while demand requiring transfers (DRT) decreases, as shown in Figure 5(c), as expected.

Because of circuitous routing and fewer routes to avoid both transfers and their penalties, the average route length $(A R L)$, which is plotted with the primary $Y$-axis in Figure 5(d), increases with increased transfer penalty. Although the number of routes (NOR) decreases, the total route length of the network (TRL) increases due to significantly increased average route length as plotted with the primary $\mathrm{Y}$-axis in the figure. Due to inefficient routing and service, total vehicle operating time in the network (TVOT), which is plotted with the secondary Y-axis, also increases with increased transfer penalty.

Overall, with increased transfer penalty, the transit networks in this example have fewer routes (Figure 5[b]), but increased total route length and increased average route length (Figure $5[\mathrm{~d}]$ ). This means that transit networks are generated as providing more circuitous routes to reduce the number of transfers in the network when higher transfer penalties are assumed. This increased circuity is also reflected in the increased adjusted degree of circuity (ADOC) in Figure 5(b). However, when demand is large enough to provide high frequencies for many routes without circuitous routing and when travel times on the links are long, direct service is preferred and the results may be substantially different.

\section{Changes in Transfer Penalties (Higher Demand Case)}

This section addresses the sensitivity analysis for the transfer penalties with a higher demand. One and a half times the previous demand and two-thirds operating speed were applied with the various transfer penalties which were applied before. The analysis results are shown in Figure 6. The same type of analysis which was shown in Figure 5 is applied, but the results are different in some aspects. 
Figure 5(a). Analysis with Different Penalites-Lower Demand Case

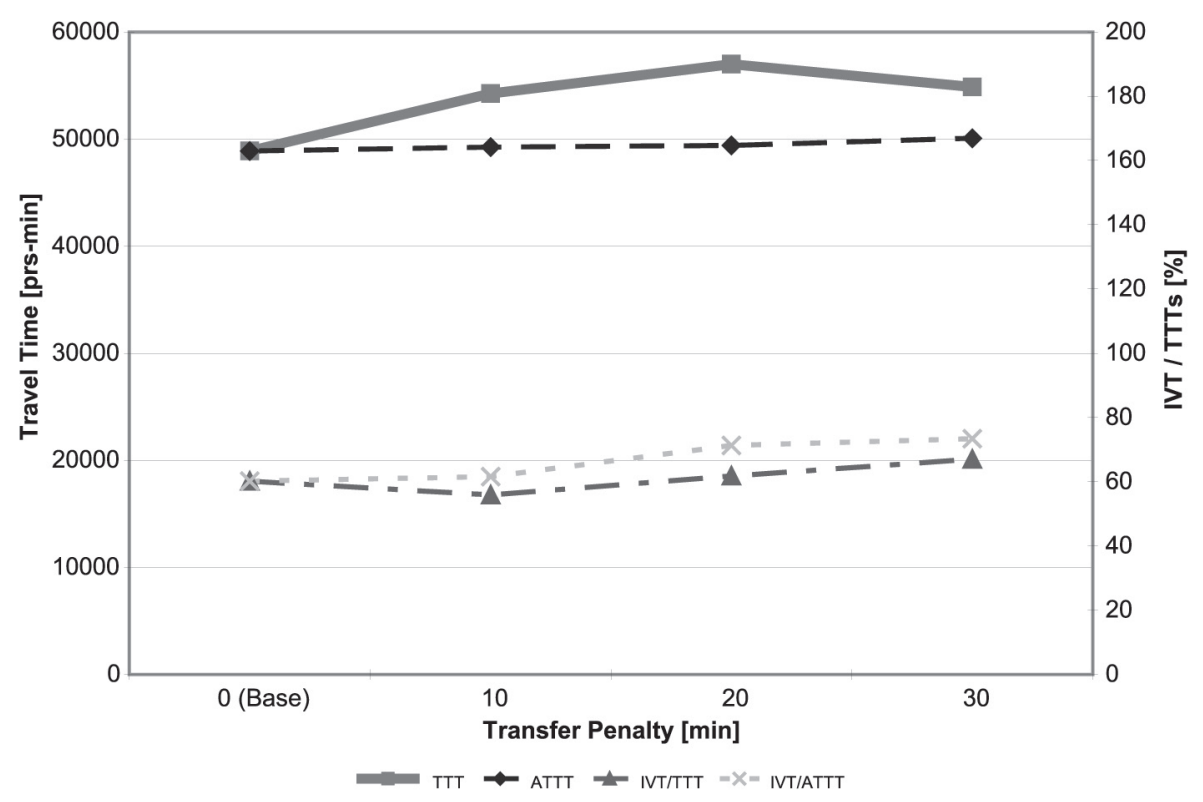

Figure 5(b)

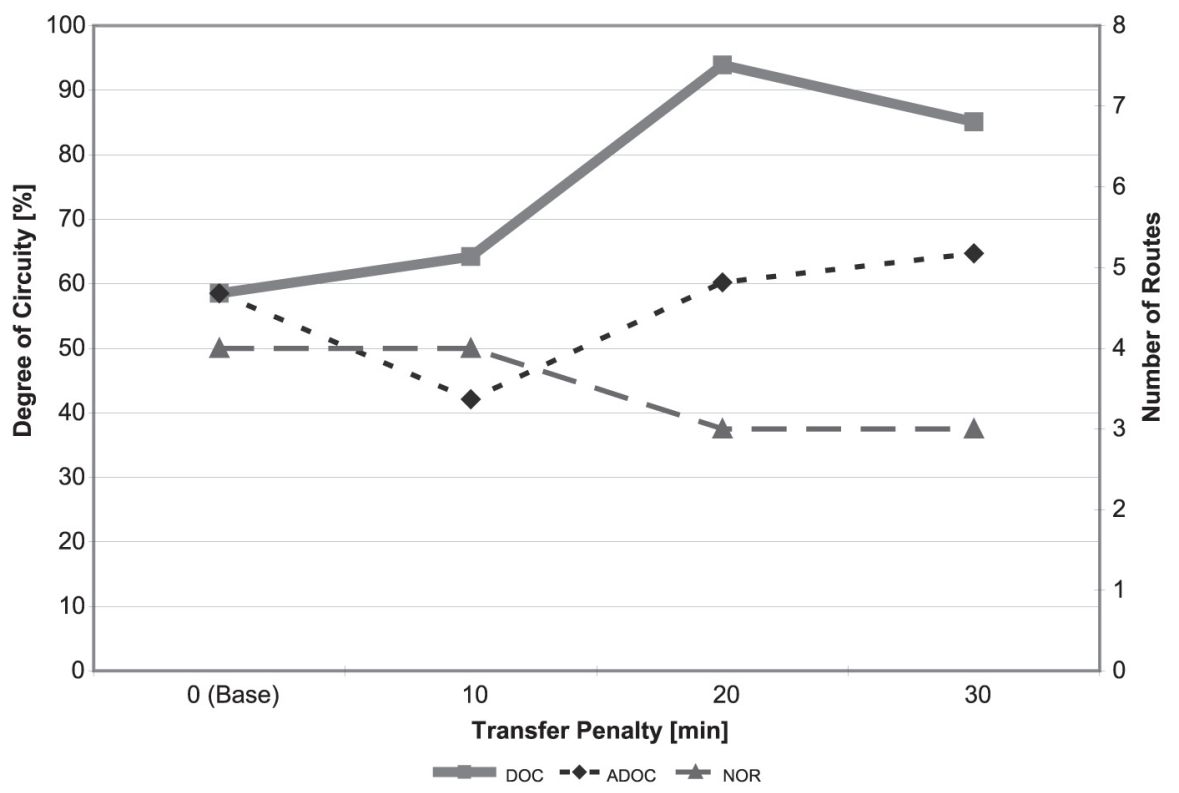


Journal of Public Transportation, Vol. 9, No. 1, 2006

Figure 5(c)

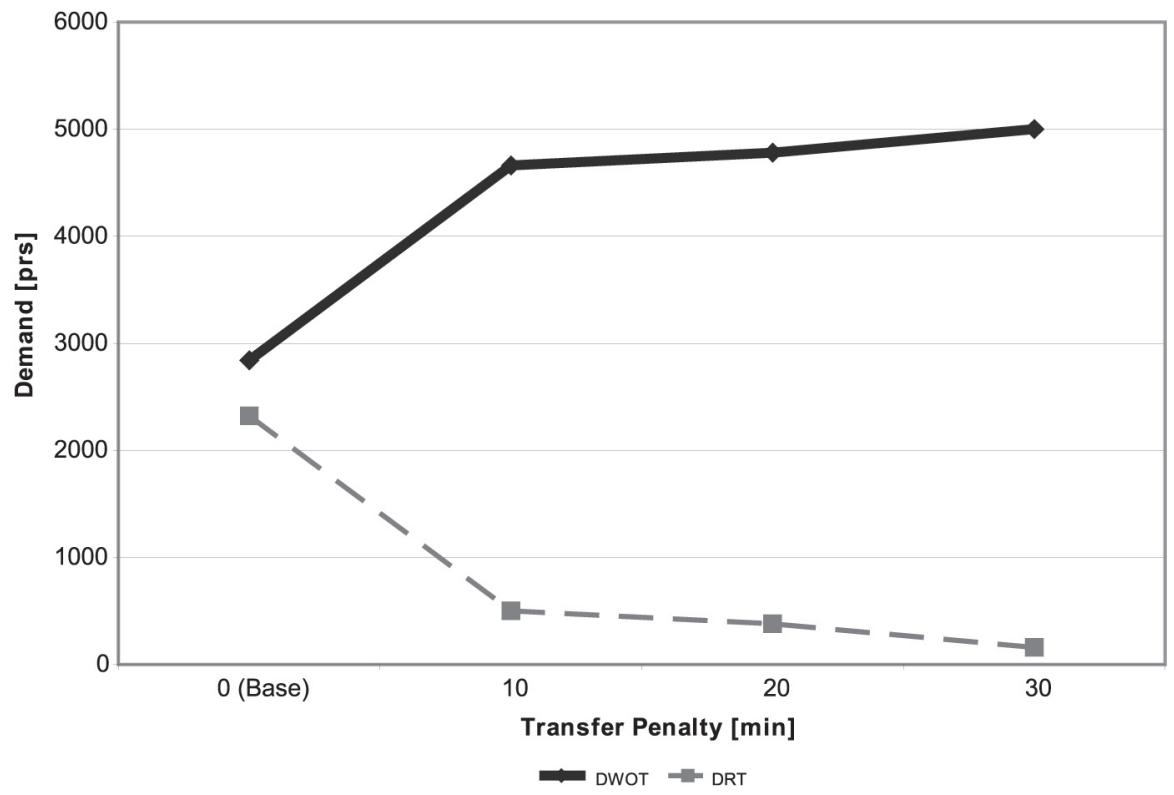

Figure 5(d)

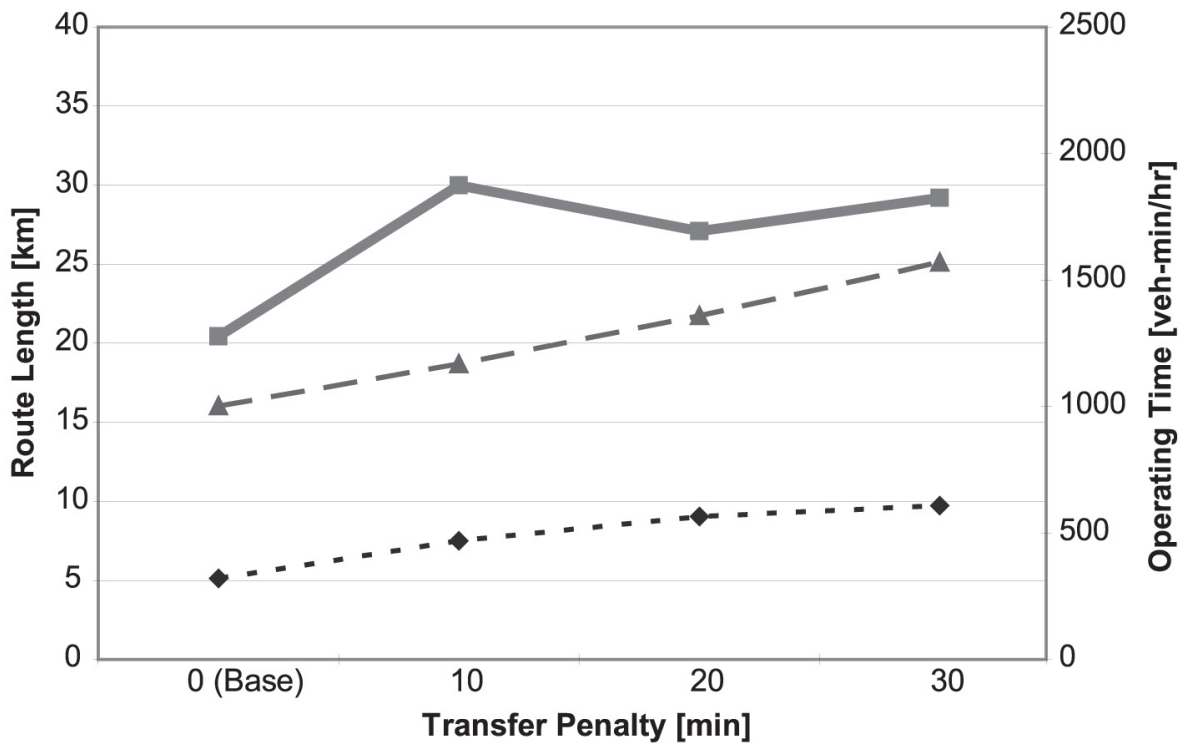

TRL $=-A R L=$ TVOT 
In Figure 6(a), total travel time in the network (TTT), which includes transfer penalties, and its adjusted version (ATTT) excluding transfer penalties are plotted with the primary $\mathrm{Y}$-axis. As shown in the figure, travel times increase with increased transfer penalties. Increased total travel time including transfer penalties is a predictable result due to the increased travel costs the transfer penalties impose. However, as before, adjusted total travel time (ATTT), which excludes the transfer penalties, also increases because the transit networks are generated inefficiently compared to the basic case to avoid transfers.

While the ratio of in-vehicle travel time to total travel time (IVT/TTT) and its adjusted version (IVT/ATTT), which are plotted with secondary $Y$-axis, increase in the lower demand range (Figure 5[a]), they decrease with increased transfer penalties in the higher demand range (Figure 6[a]). This can be explained by the fact that in the higher demand range, as transfer penalties increase, a network with more direct routes can be generated, thus reducing the percentage of in-vehicle travel time to total travel time and the number of transfers. Meanwhile, in the lower demand range, networks with circuitous routes were generated to reduce transfer penalties (TP) and waiting time (WT), thus increasing in-vehicle travel time (IVT).

In Figure 6(b), while the degree of circuity (DOC), which contains transfer penalty, increases with increased transfer penalty, its adjusted version (ADOC), which does not include transfer penalty, decreases. Because of the increased transfer penalty, users spend more total travel time (costs) including the increased transfer penalty. However, when the transfer penalty is excluded, users' combined in-vehicle travel time and transfer time decrease due to the higher number of routes in the network. The number of routes (NOR) increases with increased transfer penalty, as plotted with the secondary $Y$-axis. These results are opposite those developed for lower demand (Figure 5[b]), except for the degree of circuity. Since the degree of circuity takes into account transfer penalties, increased transfer penalties always cause an increased degree of circuity.

As shown in Figure 6(c), to avoid high transfer penalties, demand requiring transfer decreases (DRT), while direct trip demand without transfer (DWOT) increases. This is similar to the lower demand case of Figure 5(c).

In Figure 6(d), total route length (TRL) and average route length $(A R L)$ are plotted with the primary $\mathrm{Y}$-axis. With an increased number of routes to avoid transfers, the total route length in the network increases, but the average route length also increases, despite an increased number of routes (NOR). This shows that to avoid 
transfers, not only the number of routes, which represents the network extensiveness, but also the average route length, which represents circuity, increase. Due to the higher demand, more routes in the network are competitive without consolidation; however, circuitous routing is still necessary to avoid transfers as much as possible. Consequently, total vehicle operating time (TVOT), which is plotted with the secondary $Y$-axis, increases with increased transfer penalty.

Figure 6(a). Analysis with Different Transfer PenaltiesHigher Demand Case

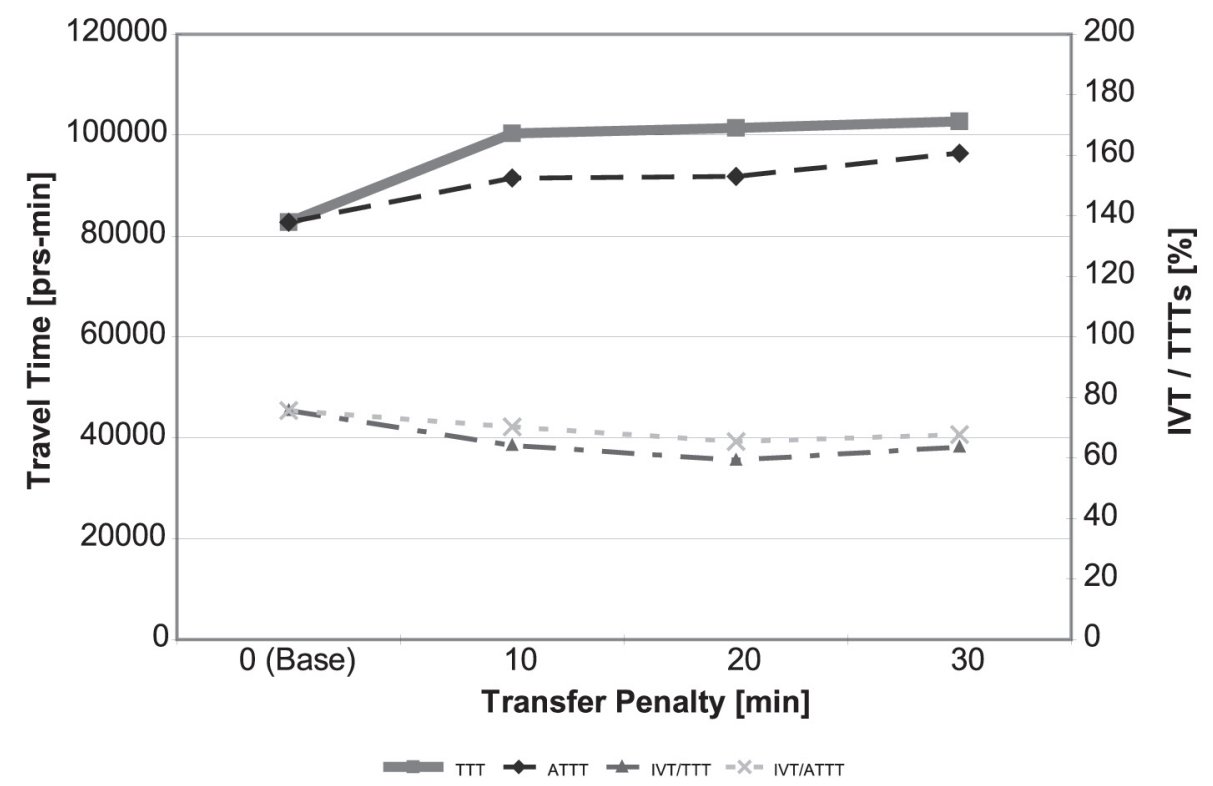




\section{Figure 6(b)}

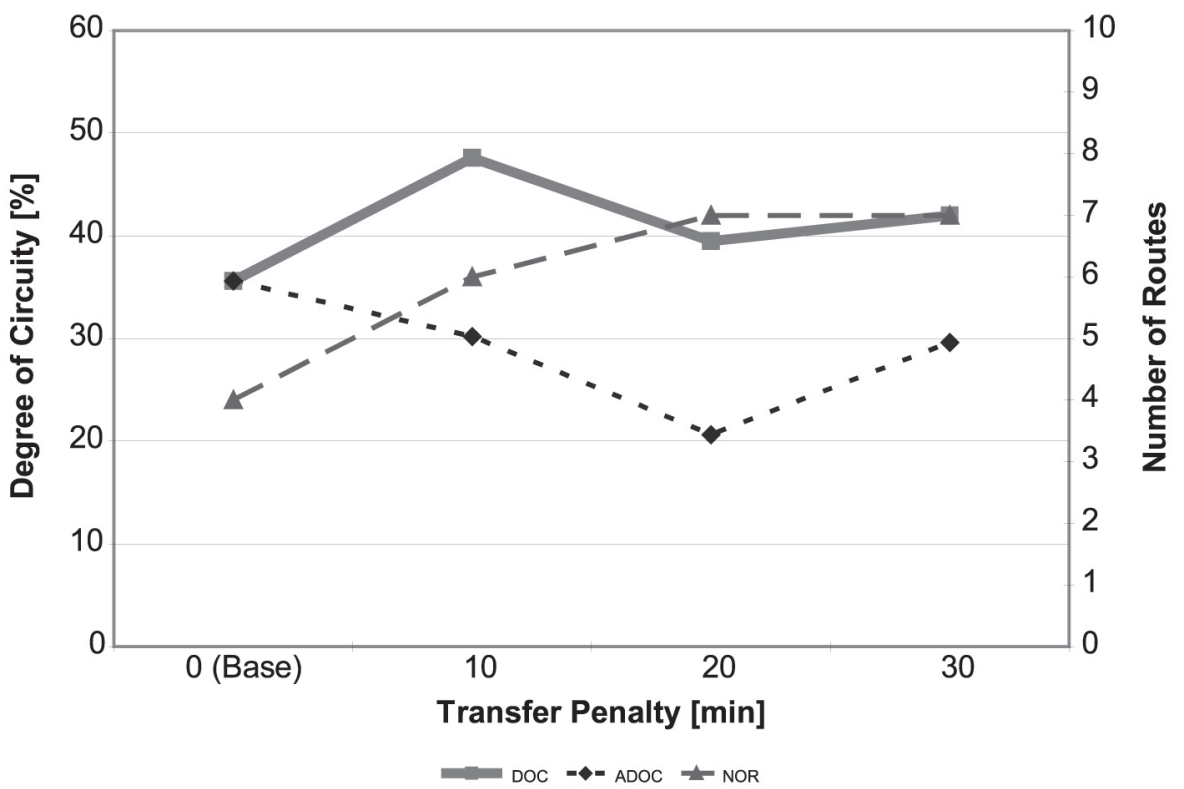

Figure 6(c)

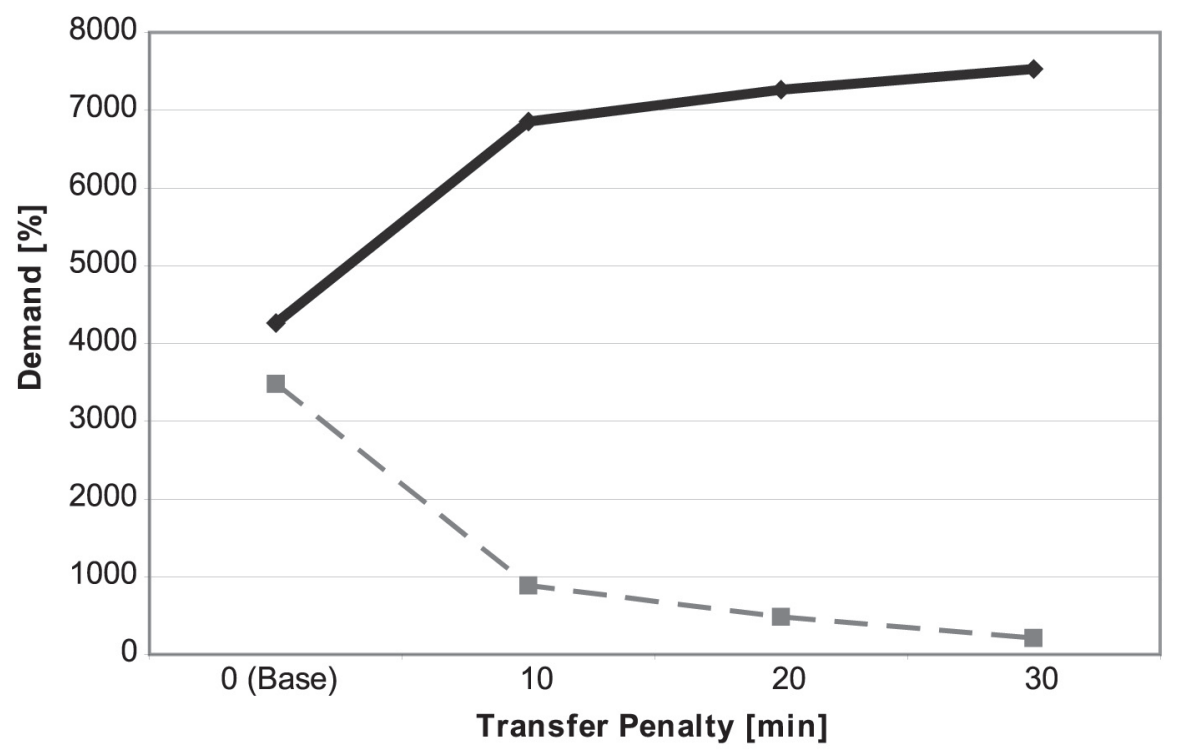

DWOT $=$ DRT 
Figure 6(d)

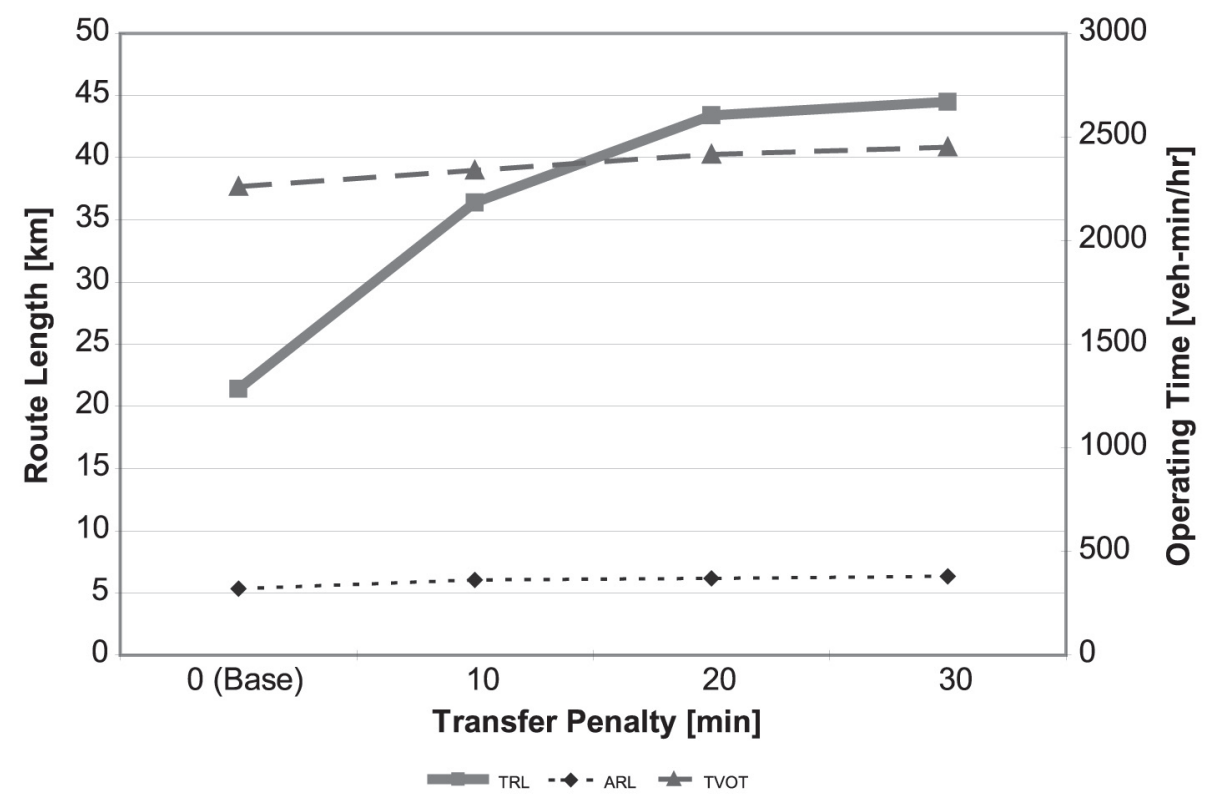

In conclusion, for different sizes of demand, the effects of transfer penalty on the network and its characteristics are defined as follows. First, with increased transfer penalties, generated networks become more inefficient both in terms of total travel time (TTT) for the users and total vehicle operating time (TVOT) for the operator. The major difference between different demand levels is the network configuration including the number of routes (NOR) and average route length $(A R L)$. If demand level is low, fewer routes are necessary to be generated with longer average route length, because not only the transfer penalty but also the waiting time should be considered. However, for the higher demand case, waiting time may not be a big concern because of higher frequencies, so a greater number of routes are generated to avoid transfers, while average route length is still increased to reduce transfers and waiting time.

\section{Three Typical Types of Transit Networks}

A few items were found to be in common from the various sensitivity analyses. First, the results matched with the logical expectation, so it is shown that TRANED can make reasonable networks. 
Second, the transit network is generated by considering and minimizing the most critical components among travel time components-in-vehicle travel time, waiting time, transfer time, and transfer penalty. Although determining a critical component is not easy, when a condition for an existing network is changed, the changed condition can be considered a critical component. In addition to taking care of the most critical component, the network affects the other components, too. For example, increased demand is directly related to decreased waiting time, but the revised network provides not only reduced waiting time, but also reduced in-vehicle travel time. In another case, increased travel times on the links (decreased operating speed) generate a revised network which requires not only increased in-vehicle travel time, but also increased other travel time, which is the sum of waiting time and transfer time.

Third, the main factor that affects the network configuration consistently is the relationship between in-vehicle travel time and waiting time. This research has shown that demand level is directly related to the changes of waiting time, and that in-vehicle travel time depends on operating speed. As shown previously, efforts to minimize total transfer penalties created networks with the lowest demand for transfers, as expected. However, two different types of networks were generated under two different demand levels because the transfer penalty is not part of the relationship between in-vehicle travel time and waiting time. On the contrary, even with different amounts of transfer penalties, increased demand still generates the same type of network, which has a higher number of routes with shorter lengths.

Fourth, under user travel time minimization, the network which consists of shorter and greater number of routes is generally more efficient for the operator in terms of total vehicle operating time in the network than one with fewer but longer routes. That is, a greater number of shorter routes can optimize vehicle operating time with more direct routing and more sectionalized frequencies.

From the above discussions, general ideas for a transit network can be extracted. Three different types of transit networks are shown in Figure 7. The first type shown in Figure 7(a) is a transfer-oriented network. This network has shorter (direct) and fewer routes with relatively high frequencies, resulting in many transfers. However, it does provides moderate in-vehicle travel time and shorter waiting time.

The transfer-avoidance network, shown in Figure $7(b)$, consists of fewer routes with relatively high frequencies, resulting in longer average route length than that of the transfer-oriented network. The transfer-avoidance network provides fewer 


\section{Figure 7. Three Typical Types of Transit Networks}

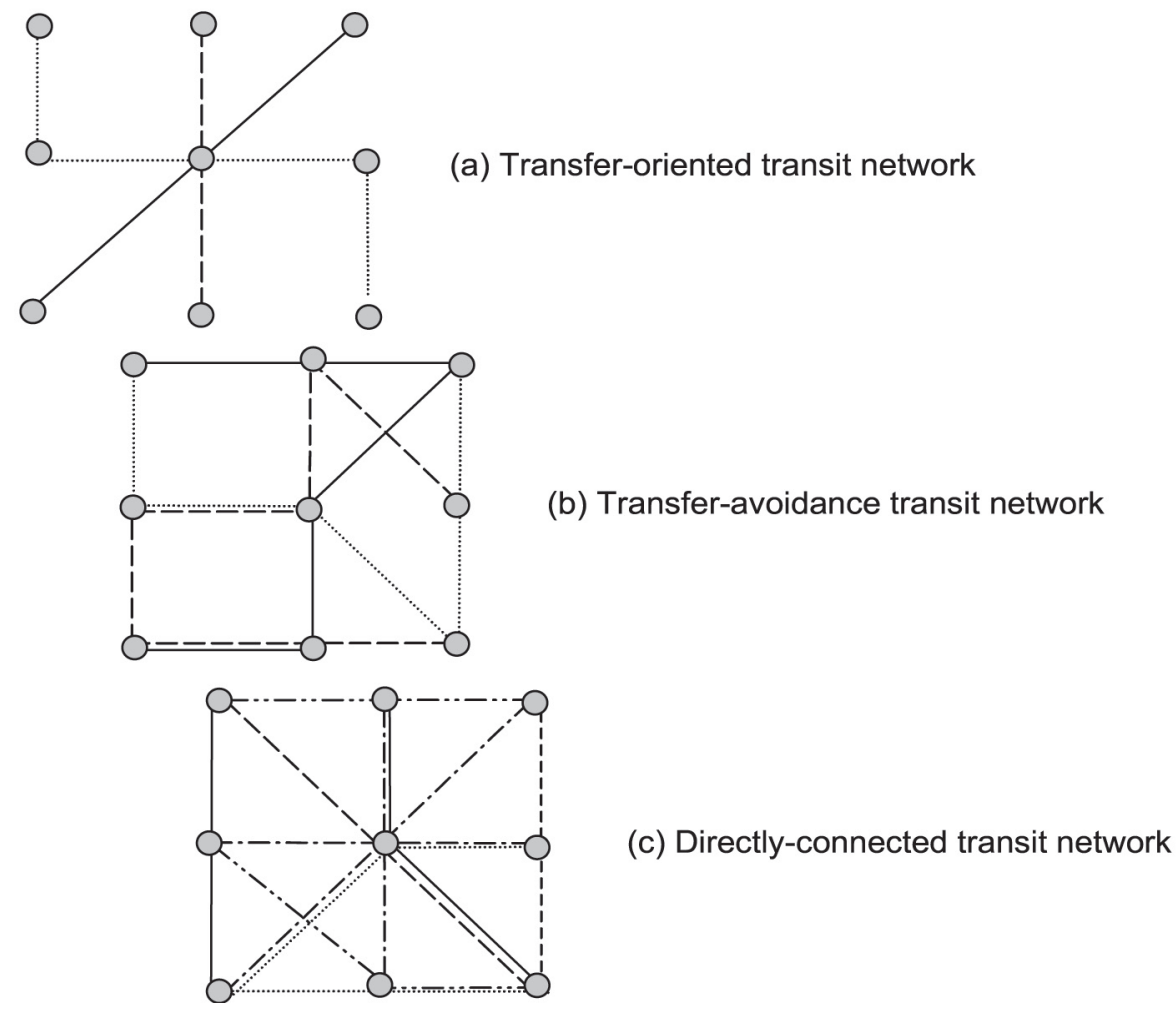

Table 2. Conceptual Relationship among Network Types and Critical Inputs

\begin{tabular}{|l|c|c|c|c|c|c|}
\hline \multirow{2}{*}{} & \multicolumn{2}{|c|}{ Demand } & \multicolumn{2}{c|}{ Travel speed } & \multicolumn{2}{c|}{$\begin{array}{c}\text { Transfer } \\
\text { penalty }\end{array}$} \\
\cline { 2 - 7 } & High & Low & High & Low & High & Low \\
\hline Transfer-oriented network & $\mathrm{O}$ & $\mathrm{O}$ & $\mathrm{O}$ & - & $\mathrm{X}$ & $\mathrm{O}$ \\
\hline Transfer-avoidance network & $\mathrm{X}$ & $\mathrm{O}$ & - & $\mathrm{X}$ & $\mathrm{O}$ & - \\
\hline Directly-connected network & $\mathrm{O}$ & $\mathrm{X}$ & $\mathrm{O}$ & $\mathrm{O}$ & $\mathrm{O}$ & $\mathrm{O}$ \\
\hline
\end{tabular}

$\mathrm{O}=$ good or adequate

$\mathrm{X}=$ inferior or incompatible

- = no strong relationship 
transfers due to longer and more circuitous routes. It also provides less transfer time and less waiting time due to higher frequencies. However, more in-vehicle travel time due to more circuitous and longer routes is required instead.

The directly-connected network, shown in Figure $7(\mathrm{c})$, consists of a greater number of routes with shorter lengths. It provides the shortest in-vehicle travel time of all three types of networks and fewer transfers due to the greater number of directlyconnected routes. However, it requires the longest waiting time of all three types because of relatively lower frequencies.

The type of network is dictated by a combination of three critical inputs-demand level, travel time on link (transit speed) and transfer penalty. Their relationships based on analysis in this research are summarized in Table 2.

As shown in the table, when the demand is high enough to provide a directly-connected network, the first or third type of network should be generated. If a high transfer penalty is added, the transfer-avoidance network and the directly-connected network become the candidates. If the demand is low and transit operating speed is also low, then the transfer-oriented network is the best choice. These relationships are conceptual, but useful when the parameters representing conditions of a city are changed.

\section{Conclusions}

The results presented in this research were quite clear and predictable, showing that Lee's model and TRANED generate reasonable transit networks and that they respond to different inputs soundly.

With increased demand, the network becomes more efficient as reflected by the improvement of all indicators. Not only waiting time decreases due to increased service frequencies, but also in-vehicle travel time decreases due to more direct services. The number of routes and passengers without transfer also increase. While the total route length and the total vehicle operating length increase, the average route length, average total travel time per trip, and average vehicle operating length per trip all decrease with increased demand.

With decreased transit operating speed, which causes increased in-vehicle travel times on the links, the generated network responds similarly to the increased demand case. Because of slower travel, the ratio of in-vehicle travel time to waiting time becomes greater, similarly, higher demand increases this same ratio through 
decreased waiting time. Thus, direct service is preferred to avoid much longer invehicle travel time due to circuitous routing. It also causes a greater number of routes with shorter average route length. The network requires longer total vehicle operating time due to increased in-vehicle travel time, but shorter vehicle operating distance due to more direct service.

In the case of increased transfer penalties, the generated transit network is less efficient to avoid them. It creates a longer network length and more circuitous routes. Consequently, increased transfer penalties result in increased total travel time and total vehicle operating time. However, as expected, demand requiring transfers decreases with increased transfer penalties.

One notable finding of these sensitivity analyses is that the characteristics of a transit network with different transfer penalties are affected substantially by the size of demand. With lower demand, to avoid transfers fewer routes with much longer lengths were generated; however, with higher demand, a greater number of routes with little increase in length were produced.

As results of the sensitivity analysis, three typical types of transit networks-transfer-oriented network, transfer-avoidance network and directly-connected network-were generated and examined. These conceptual networks can provide general ideas for changing networks with changed inputs.

The transfer-oriented network consists of shorter (direct) and fewer routes with relatively high frequencies. This type of network creates many transfers and involves transfer times. However, it provides moderate in-vehicle travel time and shorter waiting time. This type of the network is good when the transfer penalty is low and the demand is not very high.

The transfer-avoidance network consists of fewer routes with relatively high frequencies, but the average route length is longer than that of the transfer-oriented network. This type of network provides fewer transfers due to longer and more circuitous routes, and provides less transfer time and less waiting time due to higher frequencies. However, more in-vehicle travel time due to more circuitous and longer routes is required. This type of network is reasonable when the transfer penalty is high and demand is low.

The directly-connected network consists of a greater number of routes with shorter lengths. It provides the shortest in-vehicle travel time of all three types of networks and fewer transfers due to the greater number of directly-connected routes. However, it requires the longest waiting time of all three types because 
of relatively lower frequencies. The type of network is good when the demand is high enough so each route can have reasonably high frequencies. Also, this type of network is recommended when the transfer penalty is high.

\section{References}

Baaj, M. H., and Mahmassani, H. S. 1991. An Al-based approach for transit route system planning and design. Journal of Advanced Transportation 25, 2: 187210.

Ceder, A. and Israeli, Y. 1998. User and operator perspective in transit network design. Paper No. 980267, Transportation Research Board, 77th Annual Meeting, Washington, DC.

Chien, S., Yang, Z., and Hou, E. 2001. Genetic algorithm approach for transit route planning and design. Journal of Transportation Engineering 127, 3: 200-207.

Dantzig, G. B. 1967. All shortest routes in a graph. Théorie des graphes. Proceedings of the International Symposium, 91-92, Rome 1966, Dunod, Paris.

Dijkstra, E. W. 1959. A note on two problems in connection with graphs. Numerische Mathematik, 1, 269-271.

Hasselström, D. 1981. Public transportation planning, Ph.D. Dissertation, Department of Business Administration, University of Gothenburg, Sweden.

Lee, Young-Jae. 1998. Analysis and optimization of transit network design with integrated routing and scheduling, Ph.D. Dissertation, University of Pennsylvania, Philadelphia, PA.

Lee, Young-Jae, and Vuchic, V. R. 2005. Transit network design with variable demand. Journal of Transportation Engineering 131, 1: 1-10.

Mandle C. E. 1979. Evaluation and optimization of urban transportation networks. Presented at the 3rd European Congress on Operations Research, Amsterdam, Netherlands.

Newell, G. F. 1979. Some issues relating to the optimal design of bus routes. Transportation Science 13, 1.

Pattnaik, S. B., Mohan, S., and Tom, V. M. 1998. Urban bus transit network design using genetic algorithm. Journal of Transportation Engineering 124, 4: 368375. 
Rea, J. C. 1971. Designing urban transit systems: An approach to the route-technology selection problem. No. UMTA-URT-49(70)-71-6. Urban Transportation Program, University of Washington, Seattle, WA.

Shih, M, Mahmassani H. S., and Baaj, M. H. 1998. A planning and design model for transit route networks with coordinated operations. Paper No. 980418, Transportation Research Board, 77th Annual Meeting, Washington, DC.

Whiting, P. D., and Hillier, J. A. 1960. A method for finding the shortest route through a road network. Operations Research Quarterly 11: 37-40.

\section{About the Author}

YOUNG-JAE LEE (yjlee@eng.morgan.edu) is an assistant professor at the Institute for Transportation at Morgan State University. He received his Ph.D. (transportation) from the University of Pennsylvania's Department of Systems Engineering. He holds an M.S.E. transportation engineering) from the Department of Systems Engineering at the University of Pennsylvania, as well as an M.S.E. and B.S. from the Department of Civil Engineering, Urban Engineering Division, at Seoul National University, Seoul, Korea. 